TRANSACTIONS OF THE

AMERICAN MATHEMATICAL SOCIETY

Volume 364, Number 6, June 2012, Pages 3023-3070

S 0002-9947(2012)05427-4

Article electronically published on February 7, 2012

\title{
BOUNDS ON THE NUMBER AND SIZES OF CONJUGACY CLASSES IN FINITE CHEVALLEY GROUPS WITH APPLICATIONS TO DERANGEMENTS
}

\author{
JASON FULMAN AND ROBERT GURALNICK
}

\begin{abstract}
We present explicit upper bounds for the number and size of conjugacy classes in finite Chevalley groups and their variations. These results have been used by many authors to study zeta functions associated to representations of finite simple groups, random walks on Chevalley groups, the final solution to the Ore conjecture about commutators in finite simple groups and other similar problems. In this paper, we solve a strong version of the BostonShalev conjecture on derangements in simple groups for most of the families of primitive permutation group representations of finite simple groups (the remaining cases are settled in two other papers of the authors and applications are given in a third).
\end{abstract}

\section{INTRODUCTION}

One might expect that there is nothing more to be done with the study of conjugacy classes of finite Chevalley groups. For instance, over forty years ago Wall W] determined the conjugacy classes and their sizes for the unitary, symplectic, and orthogonal groups. However, the formulas involved are complicated and it is not automatic to derive upper bounds on numbers of classes or their sizes. Moreover, many applications seem to require such bounds (in particular, universal explicit bounds of the form $c q^{r}$ where $r$ is the rank of the ambient algebraic group and $q$ is the size of the field of definition). To convince the reader of this, we mention some places in the literature where bounds on the number of conjugacy classes in finite classical groups were needed:

(1) The work of Gluck Gl] on convergence rates of random walks on finite classical groups. His bounds were of the form $c q^{3 r}$.

(2) The work of Liebeck and Pyber [LiP] on number of conjugacy classes in arbitrary groups; for finite groups of Lie type their bound was $(6 q)^{r}$.

(3) The work of Maslen and Rockmore [MR on computations of Fourier transforms; they obtained a bound of $q^{n}$ for $G L(n, q)$ and $8.26 q^{n}$ for $U(n, q)$. Our bounds are actually better than this type in several senses. We prove bounds of the form $q^{r}+c q^{r-1}$ where $c$ is explicit (and absolute) for the

Received by the editors October 16, 2009 and, in revised form, July 21, 2010.

2010 Mathematics Subject Classification. Primary 20G40, $20 \mathrm{~B} 15$.

Key words and phrases. Number of conjugacy classes, simple group, Chevalley groups, partition, derangements, generating function.

The first author was partially supported by National Science Foundation grants DMS 0503901, DMS 0802082, and National Security Agency grants MDA904-03-1-004, H98230-08-1-0133.

The second author was partially supported by National Science Foundation grants DMS 0140578 and DMS 0653873.

(C)2012 American Mathematical Society Reverts to public domain 28 years from publication 
groups that are the fixed points of a Frobenius endomorphism and $d q^{r}$ for any group which has socle a Chevalley group.

(4) Liebeck and Shalev [LS1] have used bounds in the current paper to study probabilistic results about homomorphisms of certain Fuchsian groups into Chevalley groups, and random walks on Chevalley groups [LS2]. Shalev used these results in a crucial way to study the images of word maps Sh]. Our bounds were also critical in the solution of the Ore conjecture on commutators in finite simple groups [LOST].

(5) Our results have been used in studying various versions of Brauer's $k(G V)$ problem GT1 - in particular, the non-coprime version and some new related conjectures of Geoff Robinson $[\mathrm{R}$. They were used in $\mathrm{GR}$ in obtaining new results about the commuting probability in finite groups. In particular, these results will be useful in improving results of Liebeck-Pyber $[\mathrm{LiP}$ and Maróti $\mathrm{Mar}$ about the number of conjugacy classes in completely reducible linear groups over finite fields and in permutation groups.

We also use our results to prove a large part of a conjecture of Boston et al. BD. and Shalev stating that the proportion of fixed point free elements of a finite simple group in a transitive action on a finite set $X$ with $|X|>1$ is bounded away from zero. This immediately reduces to the case of primitive actions (and so to studying maximal subgroups of simple groups). This conjecture has applications to random generation of groups [FG4] and to maps between varieties over finite fields [GW].

In FG1, the conjecture was proved for Chevalley groups of bounded rank. We give another proof in this paper (Theorem 7.3 ). So we can reduce to the case of classical groups. In this paper, we prove much stronger results for all primitive actions other than when the point stabilizer is an extension field group, an imprimitive group or a subspace stabilizer. Those cases are handled in [FG2, FG3]. Here we show that in any other primitive actions, the proportion of fixed point free elements tends to 1 as the order of the classical group tends to $\infty$. Indeed, we show that the proportion of elements which are not fixed point free in any such action tends to 0 . See Theorem 7.7 .

We now state some of the main results of the paper. If $G$ is a finite group, we let $k(G)$ denote the number of conjugacy classes of $G$. See GLS for background on Chevalley groups.

Theorem 1.1. Let $G$ be a connected simple algebraic group of rank $r$ over a field of positive characteristic. Let $F$ be a Steinberg-Lang endomorphism of $G$ with $G^{F}$ a finite Chevalley group over the field $\mathbb{F}_{q}$.

(1) $q^{r}<k\left(G^{F}\right) \leq 27.2 q^{r}$.

(2) $k\left(G^{F}\right) \leq q^{r}+68 q^{r-1}$. In particular, $\lim _{q \rightarrow \infty} k\left(G^{F}\right) / q^{r}=1$ and the convergence is uniform with respect to $r$.

(3) The number of conjugacy classes of $G^{F}$ that are not semisimple is at most $68 q^{r-1}$.

(4) The number of conjugacy classes of $G$ that are $F$-stable is between $q^{r}$ and $27.2 q^{r}$.

There are much better bounds on the constants in Theorem 1.1 for many of the families. It seems likely that the correct upper bound for part (1) is about $15.2 q^{r}$ (for $S p(2 r, 2)$ ). We do obtain this bound for the simple Chevalley groups. The 27.2 comes from the argument in groups of type D in odd characteristic (this is 
because of the nature of the group of diagonal automorphisms). We give limiting values for $k\left(G^{F}\right) / q^{r}$ (as $r \rightarrow \infty$ with $q$ fixed) for each of the families of classical groups. In particular, we see that this ratio does not tend to 1 for $q$ fixed. See the tables in Section 4 for a summary of the results. There are precise formulas for the number of conjugacy classes for the exceptional Chevalley groups - see [Lu2] (and similarly, one can work out such formulas for the low rank classical groups). As we have already noted earlier in the introduction, the existence of an upper bound $k\left(G^{F}\right) \leq c q^{r}$ where $c$ depends on $r$ has already been proved (and this is straightforward). In fact, using the results of Lusztig and others about unipotent classes, it is easy to prove that $k\left(G^{F}\right)$ is bounded above by a monic polynomial in $q$ of degree $r$ (with $r$ independent of $q$ ), whence for a fixed $r$, it follows that $k\left(G^{F}\right) / q^{r} \rightarrow 1$ as $q \rightarrow \infty$. One of the key features of our result is that our bounds are of the form $c q^{r}$ or $q^{r}+c q^{r-1}$ with $c$ explicit and independent of $q, r$, and many of the applications depend on this.

We show that one can get a similar bound for almost simple Chevalley groups allowing all types of outer automorphisms.

Corollary 1.2. Let $G$ be an almost simple group with socle $S$, a Chevalley group of rank $r$ defined over $\mathbb{F}_{q}$.

(1) $k(S) \leq 15.2 q^{r}$.

(2) $k(S) \leq q^{r}+30 q^{r-1}$.

(3) $k(G) \leq 100 q^{r}$.

Recall that a permutation is called a derangement if it has no fixed points. If $G$ is a transitive group on a finite set $\Omega$, define $\delta(G, \Omega)$ to be the proportion of derangements in $G$. By an old theorem of Jordan, it follows that $\delta(G, \Omega)>0$ if $|\Omega|>1$. By a much more recent (but still elementary) theorem [CC], $\delta(G, \Omega) \geq$ $1 /|\Omega|$ for $|\Omega|>1$. See Serre [Se] for many applications of Jordan's theorem. See GW] for applications of better bounds of $\delta(G, \Omega)$ and bounds on derangements in a given coset. If $\Omega$ is the coset space $G / H$, we write $\delta(G, \Omega)$ as $\delta(G, H)$.

The classical groups are those groups related to the group of isometries $I(V)$ of a vector space $V$ over a finite field preserving some form (the 0 form, or nondegenerate Hermitian, alternating or quadratic forms). We consider any group between $I=I(V)$ and $F^{*}(I)$, where $F^{*}(I)$ is the generalized fitting subgroup of $I$. Since we are considering primitive actions, the center also acts trivially and we will often not distinguish between the linear groups and their projective versions. In particular, these are groups between $\operatorname{PSL}(V)$ and $\operatorname{PGL}(V), \operatorname{PSU}(V)$ and $\operatorname{PGU}(V)$, $P \Omega(V)$ and $\mathrm{PO}(V)$, or $\operatorname{PSp}(V)$ and $\operatorname{PCSp}(V)$.

Theorem 1.3. Let $G$ be a classical almost simple Chevalley group defined over $\mathbb{F}_{q}$ of rank $r$. Let $H$ be a maximal subgroup of $G$ that acts irreducibly and primitively on the natural module and does not preserve an extension field structure. Then there is a universal constant $\delta>0$ such that $\delta(G, H)>\delta$. Moreover, $\delta(G, H) \rightarrow 1$ as $r \rightarrow \infty$.

In fact, we prove a much stronger result than Theorem 1.3 see Theorem 7.7 See Theorem 7.3 for an analogous result for groups of bounded rank. We also prove some results about derangements in cosets of simple groups. See Section 7.

The three remaining families of maximal subgroups (reducible subgroups - including parabolic subgroups, groups preserving an extension field structure and imprimitive groups) are dealt with in [FG2, FG3]. 
The Boston-Shalev conjecture was proved for alternating and symmetric groups in [LuP. See also [D, DFG], and [FG3].

Three other results of interest that we prove and use in the preceding result are:

Theorem 1.4. Let $G$ be a connected simple algebraic group of rank $r$ of adjoint type over a field of positive characteristic. Let $F$ be a Steinberg-Lang endomorphism of $G$ with $G^{F}$ a finite Chevalley group over the field $\mathbb{F}_{q}$. There is an absolute constant A such that for all $x \in G^{F}$,

$$
\left|C_{G^{F}}(x)\right|>\frac{q^{r}}{A\left(1+\log _{q} r\right)} .
$$

See Section 6 for bounds of the form in Theorem 1.4. with explicit constants in all cases. The result holds for the finite simple Chevalley groups as well except that if $G=P S L(n, q)$ or $P S U(n, q)$, then $q^{n-1}$ needs to be replaced by $q^{n-2}$. The result also holds for orthogonal groups except that in even dimension, $q^{r}$ needs to be replaced by $2 q^{r-1}$ (but only for elements outside $S O$ ).

Theorem 1.5. Let $G$ be a finite simple Chevalley group defined over $\mathbb{F}_{q}$ of rank $r$ with $q$ a power of the prime $p$. The number of conjugacy classes of maximal subgroups of $G$ is at most $A r\left(r+r^{1 / 2} p^{3 r^{1 / 2}}+\log \log q\right)$ for some constant $A$.

In fact, we conjecture that the $r^{1 / 2} p^{3 r^{1 / 2}}$ term can be removed above. If $r$ is bounded, a much stronger result is given in [LMS.

We also prove the following result about conjugacy classes in a coset of a normal subgroup that generalizes a number of results in the literature. A special case was recently proved in $[\mathrm{BW}$. Our proof is quite short and elementary but gives a stronger result (one consequence is that the number of conjugacy classes of semisimple elements in each coset of a simple Chevalley group in the group of inner-diagonal automorphisms is the same). See Section 2 for details and for other consequences of this.

Theorem 1.6. Let $A$ be a finite group with $G$ a normal subgroup with $A / G=\langle a G\rangle$ cyclic. Let $\pi$ be any set of primes containing all prime divisors of $|A / G|$. Then the number of conjugacy classes of $\pi$-elements of $A$ in the coset $a G$ is precisely the number of $G$-conjugacy classes of $\pi$-elements in $G$ that are invariant under $A$.

We mention in particular one nice consequence of this. It follows that if $G$ is an algebraic group in characteristic $p$ with connected component $G^{0}$, then if $G^{0}$ has finitely many conjugacy classes of unipotent elements, then so does $G$. If $G^{0}$ is reductive, this is known by the work of many authors, but the passage from the connected case to the disconnected case is far from easy. See Corollary 2.6.

The organization of this paper is as follows. Section 2 studies the number of conjugacy classes in a given coset of a normal subgroup. In particular, we give a very short proof (Lemma 2.2) of a generalization of results in [BW, I] on the distribution of conjugacy classes over cosets of some normal subgroup. Section 3 obtains explicit and sharp upper bounds and asymptotics for the number of conjugacy classes in finite classical groups (some of these were announced in the survey [FG1]). This mostly involves a very careful analysis of Wall's generating functions for class numbers, but we do obtain new generating functions for groups such as $S O^{ \pm}(2 n, q)$ and $\Omega^{ \pm}(2 n, q)$ with $q$ odd. Section 4 tabulates some of the results from previous sections and summarizes corresponding results for exceptional Chevalley 
groups, due to Lübeck [L2] and others. In Section [5] we turn to almost simple groups, proving Theorem 1.1. Corollary [1.2, and some related results. Section 6 derives explicit lower bounds on centralizer sizes (and so upper bounds on the sizes of conjugacy classes) in finite classical groups. In Section 7 we get upper bounds for the number of conjugacy classes in a maximal subgroup aside from three families of maximal subgroups. We then combine those results with Theorem 6.15 to obtain Theorem [1.3, Theorem 7.7 and related results on derangements.

\section{Outer Automorphisms}

In this section, we prove some results about the number of conjugacy classes in a given coset. This will allow us to pass between various forms of our group. We first recall an elementary result of Gallagher [Ga].

Lemma 2.1. Let $H, N$ be subgroups of the finite group $G$ with $N$ normal in $G$. Then

(1) $|G: H|^{-1} k(H) \leq k(G) \leq|G: H| k(H)$; and

(2) $k(G) \leq k(N) k(G / N)$.

The following lemma will be useful in getting some better bounds for almost simple groups. See also $\mathrm{BW}, \mathrm{I}, \mathrm{K}$ for similar but somewhat weaker results.

Our method of proof is entirely different - it is shorter, more elementary and based on a very easy variant of what is known as Burnside's Lemma.

Lemma 2.2. Let $N$ be a normal subgroup of the finite group $G$ with $G / N$ cyclic and generated by $a N$. Let $\pi$ be a set of primes containing all prime divisors of $|G / N|$. Set $\alpha$ to be the number of $G$-invariant conjugacy classes of $\pi$-elements of $N$.

(1) The number of $G$-conjugacy classes of $\pi$-elements in the coset $b N$ that are a single $N$-orbit is equal to $\alpha$ for any coset $b N$.

(2) The number of $G$ conjugacy classes of $\pi$-elements in the coset aN is $\alpha$.

Proof. Note that $G$ acts on the coset $b N$ by conjugation, and thus on the $\pi$-elements in that coset. We want to calculate the number of common $G, N$ orbits on the $\pi$ elements of $b N$.

By a slight variation of Burnside's Lemma (with essentially the same proof see [FGS, §13]), this is the average number of fixed points of an element in the coset $a N$. Let $x \in a N$. Then $C_{G}(x) \cap a^{i} N=x^{i} C_{N}(x)$. Let $y=x^{e}$, where $e \equiv 1$ $\bmod [G: N]$ and $y$ is a $\pi$-element (this is possible since $\pi$ contains all prime divisors of $[G: N])$. Then $y^{i} N=x^{i} N$ for all $i$, and so $y^{i} C_{N}(x)=x^{i} C_{N}(x)$ for all $i$. If $w \in C_{N}(x) \leq C_{N}(y)$, then $y^{i} w$ is a $\pi$-element if and only if $w$ is. Thus, the number of $\pi$-elements in $C_{G}(x) \cap a^{i} N$ is the number of $\pi$-elements in $C_{N}(x)$-in particular, this number is independent of the coset. This proves (1).

If $x \in a N$, then $G=N C_{G}(x)$ and so $x^{N}=x^{G}$, whence every $G$-conjugacy class in $a N$ is a single $N$-orbit. So (1) implies (2).

This allows us to prove a generalization of part of Lemma 2.1. If $\pi$ is a set of primes and $X$ is a finite group, let $k_{\pi}(X)$ be the number of conjugacy classes of $\pi$-elements of $X$.

Lemma 2.3. Let $N$ be a normal subgroup of the finite group $G$. Let $\pi$ be a set of primes. Let $x_{i} N$ denote a set of representatives of the $\pi$-conjugacy classes of 
$G / N$. Let $f\left(x_{i}\right)$ denote the number of $N$-conjugacy classes of $\pi$-elements that are $x_{i}$-invariant. Then

$$
k_{\pi}(G) \leq \sum_{i=1}^{r} f\left(x_{i}\right) \leq k_{\pi}(G / N) k_{\pi}(N) .
$$

Proof. If $y \in G$ is a $\pi$-element, then $y N$ is conjugate to $x_{i} N$ for some $i$.

Set $G_{i}=\left\langle N, x_{i}\right\rangle$ and note that every prime divisor of $\left[G_{i}: N\right]$ is in $\pi$. By Lemma 2.2. the number of $G_{i}$ conjugacy classes of $\pi$-elements in $x_{i} N$ is at most $f\left(x_{i}\right) \leq$ $k_{\pi}(N)$. Thus, the number of $G$-conjugacy classes of $\pi$-elements that intersect $x_{i} N$ is at most $f\left(x_{i}\right)$. This completes the proof.

Corollary 2.4. Let $N$ be a normal subgroup of $G$ with $\pi$ a set of primes containing all prime divisors of $|G / N|$. If each $\pi$-element $g$ of $G$ satisfies $G=N C_{G}(g)$, then $G / N$ is abelian and the number of $\pi$-conjugacy classes of $G$ in any coset of $N$ is $k_{\pi}(N)$.

Proof. We first show that $G / N$ is abelian. Consider a coset $x N$. Let $x \in G$. Then $x N=y N$, where $y$ is a $\pi$-element (as in the previous proof). Then $[y, G]=$ $\left[y, N C_{G}(y)\right] \leq N$. Hence $[G, G] \leq N$, and so $G / N$ is abelian.

The hypothesis implies that every $\pi$-class of $G$ is a single $N$-orbit. Applying Lemma 2.2 to the subgroup $H:=\langle N, x\rangle$ shows that the number of common $H, N$ orbits on $\pi$-elements of $x N$ is the number of common $H, N$-orbits on $\pi$-elements in $N$. The hypothesis implies that all $G$-orbits on $\pi$-elements are $N$-orbits, whence the number of conjugacy classes of $\pi$-elements in $x N$ is $k_{\pi}(N)$.

The hypotheses apply to the case where $N$ is a simple Chevalley group in characteristic $p$, and $G$ is contained in the group of inner-diagonal automorphisms on $N$ with $\pi$ consisting of all primes other than $p$. See [S1, 2.12]. Indeed, the same applies to a slightly more general situation.

Corollary 2.5. Let $N$ be a perfect Chevalley group with $N / Z(N)$ simple. Assume that $N$ is normal in $G, G$ induces inner-diagonal automorphisms of $N$ and $C_{G}(N)=Z(G)$ has order prime to $p$. Then the number of semisimple conjugacy classes in each coset of $S$ in $G$ is the same.

For example, the previous result applies to $\mathrm{SL}(n, q)<\mathrm{GL}(n, q)$.

Another easy consequence of Lemma 2.2 is showing that the finiteness of unipotent classes in a disconnected reductive group follows from the result for connected reductive groups. There have been several proofs of the finiteness of the number of unipotent classes in the connected case - see [Lus1. The result is also known for the disconnected case (see $\mathrm{Gu}$ ] for a generalization).

Corollary 2.6. Let $G$ be an algebraic group defined over a finite field $L$ of characteristic $p$. Let $H$ be its connected component. Suppose that $H$ has finitely many conjugacy classes of unipotent elements. Then $G$ has finitely many conjugacy classes of unipotent elements.

Proof. Let $U$ be the variety of unipotent elements in $G$. So $U$ is defined over $L$. Let $k$ be the algebraic closure of $L$. Suppose that $H$ has $m$ conjugacy classes of unipotent elements. Let $L^{\prime} / L$ be a finite extension. By Lang's theorem, $H\left(L^{\prime}\right)$ 
has at most me conjugacy classes of unipotent elements, where $e$ is the maximal number of connected components in $C_{H}(u)$ for $u$ a unipotent element of $H$.

Let $s$ be the number of conjugacy classes of $p$-elements in $G / H$ (which is isomorphic to $\left.G\left(L^{\prime}\right) / H\left(L^{\prime}\right)\right)$. By Lemma 2.3, $G\left(L^{\prime}\right)$ has at most sme conjugacy classes of p-elements.

Since $G(k)$ is the union of the $G\left(L^{\prime}\right)$ as $L^{\prime}$ ranges over all finite extensions of $L^{\prime} / L$, it follows that $G(k)$ has at most sme conjugacy classes of unipotent elements.

By [GLMS, Prop 1.1], it follows that the number of unipotent classes of $G$ is the same as the number of $G(k)$ classes of unipotent elements.

The following must surely be known, but it follows easily from Lemma 2.2 .

Corollary 2.7. Let $m>3$. Then $k\left(A_{m}\right)<k\left(S_{m}\right)$.

Proof. Let $a$ be the number of $A_{m}$ classes that are stable under $S_{m}$. Let $b$ be the number of $S_{m}$ classes in $A_{m}$ that are not $A_{m}$ classes. Clearly $k\left(A_{m}\right)=a+2 b$ and, by Lemma 2.2. $k\left(S_{m}\right)=2 a+b$. So we only need to show that $a>b$. If $m=4,5$, the result is clear. So we show that $a>b$ for $m>5$.

Note that $b$ is precisely the number of classes where all cycle lengths are distinct and odd. There clearly is an injection into stable classes; namely, since $m>4$ the largest cycle is odd of length $j \geq 5$, so one can replace it by a product of two 2 -cycles and $j-4$ fixed points. The image misses an element of order 4 , and so the injection is not surjective.

Another easy consequence of Lemma 2.2 is:

Corollary 2.8. Let $N$ be a normal subgroup of the finite group $G$. Let $K$ be a subgroup of $G$ containing $N$ with $K / N$ cyclic and central in $G / N$. Let $\pi$ be a set of primes containing all prime divisors of $|K / N|$. Let $\Delta$ be the set of $G$-conjugacy classes of $\pi$-elements such that $K=N C_{G}(g)$. Then $\Delta$ is equally distributed among the cosets of $N$ contained in $K$.

Proof. Let $\Gamma$ be the union of the conjugacy classes in $\Delta$. Note that $g \in \Gamma$ implies that $g \in K$.

Let $\alpha$ be the number of $K$-stable conjugacy classes of $\pi$-elements of $N$.

By the proof of Lemma 2.2, it follows that $K$ has precisely $\alpha$ orbits on $\Gamma \cap g N$ for each $g \in K$. Since $G / K$ acts freely on the $K$ orbits on $\Gamma$, it follows that there are precisely $\alpha /[G: K]$ elements of $\Delta$ in each coset of $K / N$.

In certain cases, one can describe the conjugacy classes in a coset very nicely using the Shintani correspondence. See $[\mathrm{K}, \S 2]$. We first need some notation. Let $G$ be a connected algebraic group. Let $F$ be a Lang-Steinberg endomorphism of $G$ (i.e. the fixed points $G^{F}$ form a finite group). We first recall the well-known result of Lang-Steinberg.

Lemma 2.9. Let $G$ be a connected linear algebraic group, and let $F$ be a surjective endomorphism of $G$ such that $G^{F}$ is finite. Then the map $f: x \mapsto x^{-F} x$ from $G$ to $G$ is surjective.

Note that if $F$ is such an endomorphism of a simple connected algebraic group $G$, then we can attach a prime power $q=q_{F}$ of the characteristic to $F$. Then $G^{F}$ is said to be defined over $q$. We write $G^{F}=G(q)$ (of course, there may be more 
than one endomorphism associated with the same $q$ - in particular, this is the case if $G$ admits a graph automorphism). The Shintani correspondence is:

Theorem 2.10. Let $G$ be a connected linear algebraic group with Frobenius map $F$. Let $H$ be the fixed points of $F^{m}$. We view $F$ as an automorphism of $H$ of order $m$. Then there is a bijection $\psi$ between conjugacy classes in the coset $F H$ and conjugacy classes in $H^{F}$. Moreover, $C_{H}(F h) \cong C_{H^{F}}(k)$, where $\psi[F h]=[k]$.

We sketch the proof (see $[\mathbf{S}$ ). We may define $\psi$ as follows. Given $x$ in $H$, let $\alpha_{x}$ be such that $\alpha_{x}^{-F} \alpha_{x}=x$. Define $N(x)=x^{F^{m-1}} \cdots x^{F^{2}} x^{F} x$. Set $\psi(F x)=$ $\alpha_{x} N(x) \alpha_{x}^{-1} \in H^{F}$. This depended upon the choice of $\alpha_{x}$, but another choice preserves the conjugacy class and $\psi$ defines the desired bijection on classes. It is straightforward to see that this bijection has the properties described in the theorem.

Combining this theorem together with Lemma 2.2 gives:

Corollary 2.11. Let $G$ be a connected linear algebraic group with Frobenius map $F$. Let $H$ be the fixed points of $F^{m}$. We view $F$ as an automorphism of $H$ of order $m$. Then $k\left(H^{F}\right)$ is equal to the number of $H$-conjugacy classes in the coset $F H$ and is also equal to the number of $F$-stable conjugacy classes in $H$.

Proof. The previous theorem implies that the first two quantities are equal. Lemma 2.2 implies that the second and third quantities are equal.

\section{NUMBER OF CONJUGACY CLASSES IN ClASSICAL GROUPS}

In this section, we obtain upper bounds for $k(G)$ with $G$ a classical group. Subsection 3.1 develops some preliminary tools. Type A groups are treated in Subsections 3.2 and 3.3. symplectic and orthogonal groups are treated in Subsections 3.4 and 3.5 respectively.

3.1. Preliminaries. The following result of Steinberg [St1, 14.8, 14.10] gives lower bounds for $k(G)$ with $G$ a Chevalley group. See also [C, p. 102]. The inequality is not stated explicitly though.

Theorem 3.1. Let $G$ be a connected reductive group of semisimple rank $r>0$. Let $F$ be a Frobenius endomorphism of $G$ associated to $q$. Let $Z_{0}$ denote the connected component of the center of $G$. The number of semisimple conjugacy classes in $G^{F}$ is at least $\left|Z_{0}^{F}\right| q^{r}$ with equality if $G^{\prime}$ is simply connected. In particular, $k\left(G^{F}\right)>q^{r}$.

Proof. By [St1, 14.8], the number of $F$-stable conjugacy classes is exactly $\left|Z_{0}^{F}\right| q^{r}$. If $G^{\prime}$ is simply connected, then the centralizer of any semisimple element is connected, whence there is a bijection between stable conjugacy classes of semisimple elements and semisimple conjugacy classes in $G^{F}$.

On the other hand, every $F$-stable class has a representative in $G^{F}$ by St1, 14.10], and so there are at least $q^{r}$ semisimple conjugacy classes in $G^{F}$ (with equality in the simply connected case). Since there must be at least 1 stable class of nontrivial unipotent elements, the last statement follows.

The following two asymptotic lemmas will be useful.

Lemma 3.2 (Darboux [O]). Suppose that $f(u)$ is analytic for $|u|\langle r, r\rangle 0$ and has a finite number of simple poles on $|u|=r$. Let $w_{j}$ denote the poles, and suppose 
that $f(u)=\sum_{j} \frac{g_{j}(u)}{1-u / w_{j}}$ with $g_{j}(u)$ analytic near $w_{j}$. Then the coefficient of $u^{n}$ in $f(u)$ is

$$
\sum_{j} \frac{g_{j}\left(w_{j}\right)}{w_{j}^{n}}+o\left(1 / r^{n}\right) .
$$

Lemma $3.3([\mathrm{O})$. Suppose that $f(u)$ is analytic for $|u|<R$. Let $M(r)$ denote the maximum of $|f|$ restricted to the circle $|u|=r$. Then for any $0<r<R$, the coefficient of $u^{n}$ in $f(u)$ has absolute value at most $M(r) / r^{n}$.

The following lemma is Euler's pentagonal number theorem (see for instance page 11 of [A1]).

Lemma 3.4. For $q>1$,

$$
\begin{aligned}
\prod_{n=1}^{\infty}\left(1-\frac{1}{q^{i}}\right) & =1+\sum_{n=1}^{\infty}(-1)^{n}\left(q^{-\frac{n(3 n-1)}{2}}+q^{-\frac{n(3 n+1)}{2}}\right) \\
& =1-q^{-1}-q^{-2}+q^{-5}+q^{-7}-q^{-12}-q^{-15}+\cdots .
\end{aligned}
$$

Throughout this section quantities which can be easily re-expressed in terms of the infinite product $\prod_{i=1}^{\infty}\left(1-\frac{1}{q^{i}}\right)$ will often arise, and Lemma 3.4 gives arbitrarily accurate upper and lower bounds on these products. Hence we will state bounds like $\prod_{i=1}^{\infty}\left(1+\frac{1}{2^{i}}\right)=\prod_{i=1}^{\infty} \frac{\left(1-\frac{1}{4^{i}}\right)}{\left(1-\frac{1}{2^{i}}\right)} \leq 2.4$ without explicitly mentioning Euler's pentagonal number theorem on each occasion.

3.2. $G L(n, q)$ and its relatives. To begin we discuss $G L(n, q)$. By a formula of Feit and Fine [FF, $\mathrm{M} 1$, the number of conjugacy classes in $G L(n, q)$ is the coefficient of $t^{n}$ in the generating function

$$
\prod_{i=1}^{\infty} \frac{1-t^{i}}{1-q t^{i}}
$$

Using clever reasoning and Euler's pentagonal number theorem, it is proved in $\mathrm{MR}$ ] that the number of conjugacy classes of $G L(n, q)$ is less than $q^{n}$.

To this we add the following simple proposition.

Proposition 3.5. (1) For $q$ fixed, $\lim _{n \rightarrow \infty} \frac{k(G L(n, q))}{q^{n}}=1$.

(2) $q^{n}-q^{n-1} \leq k(G L(n, q)) \leq q^{n}$. Thus $\lim _{q \rightarrow \infty} \frac{k(G L(n, q))}{q^{n}}=1$, and the convergence is uniform in $n$.

Proof. The generating function for conjugacy classes of $G L(n, q)$ gives that $\frac{k(G L(n, q))}{q^{n}}$ is the coefficient of $t^{n}$ in

$$
\frac{1}{1-t} \prod_{i \geq 1} \frac{1-t^{i} / q^{i}}{1-t^{i+1} / q^{i}}
$$

Note that the function $g(t)=\prod_{i \geq 1} \frac{1-t^{i} / q^{i}}{1-t^{i+1} / q^{i}}$ is analytic for $|t|<q^{1 / 2}$. (This follows from a basic fact in analysis that absolute convergence of the product $\prod_{i}\left(1+a_{i}\right)$ is equivalent to convergence of the sum $\left.\sum_{i}\left|a_{i}\right|\right)$. Applying Lemma 3.2 with $f(t)=g(t) /(1-t)$ and $w_{j}=1$ proves the first assertion. For the second assertion, the upper bound on $k(\mathrm{GL}(n, q))$ was mentioned earlier, and the lower 
bound holds since GL $(n, q)$ has $q^{n}-q^{n-1}$ semisimple conjugacy classes (the similarity class of a semisimple element in $\operatorname{GL}(n, q)$ is determined by its characteristic polynomial and clearly the number of monic polynomials with non-zero constant term is $\left.q^{n}-q^{n-1}\right)$.

Remark. In fact, $\frac{k(G L(n, q))}{q^{n}}$ is even closer to 1 than one might suspect from Proposition 3.5 . Indeed,

$$
\frac{1}{1-t} \prod_{i \geq 1} \frac{1-t^{i} / q^{i}}{1-t^{i+1} / q^{i}}-\frac{1}{1-t}
$$

is analytic for all $|t|<q^{1 / 2}$ (subtracting the $(1-t)^{-1}$ removed the pole at $t=1$ ). Thus Lemma 3.3 gives that for any $0<\epsilon<1 / 2,\left|\frac{k(G)}{q^{n}}-1\right| \leq \frac{C_{q, \epsilon}}{q^{n(1 / 2-\epsilon)}}$, where $C_{q, \epsilon}$ is a constant depending on $q$ and $\epsilon$ (which one could make explicit with more effort). This is consistent with the fact $([\overline{\mathrm{BFH}}], \underline{\mathrm{MR}}])$ that $k(\mathrm{GL}(n, q))$ is a polynomial in $q$ with lead term $q^{n}$ and vanishing coefficients of $q^{n-1}, \cdots, q^{\left\lfloor\frac{n+1}{2}\right\rfloor}$.

Macdonald M1] derived formulas for the number of conjugacy classes of $S L(n, q)$, $\operatorname{PGL}(n, q)$ and $\operatorname{PSL}(n, q)$ in terms of $k(G L(n, q))$. As these will be used below it is useful to recall them. Let

$$
\phi_{r}(n)=n^{r} \prod_{p \mid n}\left(1-p^{-r}\right)
$$

where the product is over primes dividing $n$. Thus $\phi_{1}(n)$ is Euler's $\phi$ function. Macdonald showed that

$$
\begin{aligned}
k(S L(n, q)) & =\frac{1}{q-1} \sum_{d \mid n, q-1} \phi_{2}(d) k(G L(n / d, q)), \\
k(P G L(n, q)) & =\frac{1}{q-1} \sum_{d \mid n, q-1} \phi_{1}(d) k(G L(n / d, q)),
\end{aligned}
$$

and

$$
k(P S L(n, q))=\frac{1}{(q-1) \operatorname{gcd}(n, q-1)} \sum_{d_{1}, d_{2}} \phi_{1}\left(d_{1}\right) \phi_{2}\left(d_{2}\right) k\left(G L\left(\frac{n}{d_{1} d_{2}}, q\right)\right),
$$

where the sum is over all pairs of divisors $d_{1}, d_{2}$ of $q-1$ such that $d_{1} d_{2}$ divides $n$.

Proposition 3.6. (1) $q^{n-1}<k(S L(n, q)) \leq 2.5 q^{n-1}$.

(2) $k(S L(n, q)) \leq q^{n-1}+3 q^{n-2}$. Thus $\lim _{q \rightarrow \infty} \frac{k(S L(n, q))}{q^{n-1}}=1$, and the convergence is uniform in $n$.

(3) For $q$ fixed, $\lim _{n \rightarrow \infty} \frac{k(S L(n, q))}{q^{n-1}}=\frac{1}{1-1 / q}$.

Proof. The lower bound holds by Theorem 3.1. From Macdonald's formula for $k(S L(n, q))$ one checks that $k(S L(2, q)) \leq q+4$ and $k(S L(3, q)) \leq q^{2}+q+8$ (and that parts (1) and (2) hold for $q=2,3$ and $n \leq 4)$. So assume that $n \geq 4$. 
If $q=2$, then the upper bound in part (1) follows by [MR Lemma A.1]. So assume also that $q \geq 3$. Since $k(G L(n / d, q)) \leq q^{n / d}$, it follows that

$$
\begin{aligned}
k(S L(n, q)) & \leq \frac{1}{q-1} \sum_{d \mid n, q-1} d^{2} q^{n / d} \\
& \leq \frac{1}{q-1}\left[q^{n}+(q-1)^{2}\left(q^{n / 2}+q^{n / 2-1}+\cdots\right)\right] \\
& \leq q^{n} /(q-1)+q^{n / 2+1} .
\end{aligned}
$$

For $q \geq 3, n \geq 4$, this is easily seen to be at most $2.5 q^{n-1}$.

The upper bound in part (2) when $q=2$ follows from [MR, Lemma A.1]. For $q \geq 3, n \geq 6$ one has that

$$
q^{n} /(q-1)+q^{n / 2+1} \leq q^{n-1}+3 q^{n-2},
$$

and one easily checks from Macdonald's formula that $k(S L(n, q)) \leq q^{n-1}+3 q^{n-2}$ for $q \geq 3, n \leq 5$.

For part (3), note from Macdonald's formula that

$$
\frac{k(S L(n, q))}{q^{n-1}}=\frac{1}{1-1 / q} \sum_{d \mid n, q-1} \phi_{2}(d) \frac{k(G L(n / d, q))}{q^{n}} .
$$

Since $q$ is fixed, it is clear from Proposition 3.5 that only the $d=1$ term contributes in the $n \rightarrow \infty$ limit, yielding the result.

The following corollary concerns groups between $S L(n, q)$ and $G L(n, q)$ or between $P S L(n, q)$ and $P G L(n, q)$.

Corollary 3.7. (1) Suppose that $S L(n, q) \subseteq H \subseteq G L(n, q)$, and let $j$ denote the index of $H$ in $G L(n, q)$. Then

$$
\frac{q^{n-1}(q-1)}{j} \leq k(H) \leq \frac{q-1}{j} k(S L(n, q)) \leq \frac{q^{n}+3 q^{n-1}}{j} .
$$

(2) Suppose that $P S L(n, q) \subseteq H \subseteq P G L(n, q)$, and let $j$ denote the index of $H$ in $P G L(n, q)$. Then

$$
\frac{q^{n-1}}{j} \leq k(H) \leq \frac{\operatorname{gcd}(n, q-1)}{j} k(P S L(n, q)) \leq \frac{q^{n-1}+5 q^{n-2}}{j} .
$$

Proof. Let $H$ be as in part (1) of the corollary. Then $k(H) \geq \frac{k(G L(n, q))}{j} \geq \frac{q^{n-1}(q-1)}{j}$, where the first inequality is Lemma 2.1 and the second is the fact that $G L(n, q)$ has $q^{n-1}(q-1)$ semisimple conjugacy classes. The inequality $k(H) \leq \frac{q-1}{j} k(S L(n, q))$ comes from Lemma 2.1, and Proposition 3.6 yields the inequality $(q-1) k(S L(n, q))$ $\leq q^{n}+3 q^{n-1}$.

Let $H$ be as in part (2) of the corollary. Then $k(H) \geq \frac{k(P G L(n, q))}{j} \geq \frac{q^{n-1}}{j}$, where the first inequality is Lemma 2.1 and the second is the fact that $P G L(n, q)$ has at least $q^{n-1}$ conjugacy classes (clear from Macdonald's formula and the fact that $G L(n, q)$ has at least $q^{n-1}(q-1)$ conjugacy classes). The inequality $k(H) \leq$ $\frac{\operatorname{gcd}(n, q-1)}{j} k(P S L(n, q)) \quad$ comes from Lemma 2.1 and the inequality $\operatorname{gcd}(n, q-1) k(P S L(n, q)) \leq q^{n-1}+5 q^{n-2}$ follows from Macdonald's formula for $k(P S L(n, q))$ and an analysis similar to that in Proposition 3.6. 
We close this section with the following exact formula for the number of conjugacy classes of a group $H$ between $S L(n, q)$ and $G L(n, q)$. It involves the quantity $\phi_{2}$ defined earlier in this section.

Proposition 3.8. Suppose that $S L(n, q) \subseteq H \subseteq G L(n, q)$ and let $j$ denote the index of $H$ in $G L(n, q)$. Then

$$
k(H)=\frac{1}{j} \sum_{d \mid(j, n)} \phi_{2}(d) k\left(G L\left(\frac{n}{d}, q\right)\right) .
$$

Proof. As in M1, to each conjugacy class of $G L(n, q)$ there is associated a partition $\nu$ of $n$. To describe this recall that conjugacy classes of $G L(n, q)$ are parametrized by associating to each monic irreducible polynomial $p(x)$ over $F_{q}$ with non-zero constant term a partition; if the partition corresponding to $p(x)$ has $m_{i}$ parts of size $i$, then it contributes $\operatorname{deg}(p) m_{i}$ parts of size $i$ to the partition $\nu$. Throughout the proof we let $c_{\nu}$ denote the number of conjugacy classes of $G L(n, q)$ of type $\nu$. We also let $\nu_{1}, \cdots, \nu_{r}$ denote the parts of $\nu$.

Given the partition $\nu$, we determine the number of conjugacy classes of $G L(n, q)$ of type $\nu$ in $H$, multiply it by the number of $H$ classes into which each such class splits (this number depends only on $\nu$ ) and then sum over all $\nu$. Arguing as on pages 33-36 of [M1] shows that the number of conjugacy classes of $G L(n, q)$ of type $\nu$ in $H$ is

$$
\frac{\operatorname{gcd}\left(j, \nu_{1}, \cdots, \nu_{r}\right) c_{\nu}}{j}
$$

and that each such class splits into $\operatorname{gcd}\left(j, \nu_{1}, \cdots, \nu_{r}\right)$ many $H$ classes. Thus the total number of conjugacy classes of $H$ is

$$
\frac{1}{j} \sum_{|\nu|=n} \operatorname{gcd}\left(j, \nu_{1}, \cdots, \nu_{r}\right)^{2} c_{\nu}
$$

Arguing as on pages 36-37 of M1, this can be rewritten as

$$
\frac{1}{j} \sum_{d \mid(j, n)} \phi_{2}(d) k\left(G L\left(\frac{n}{d}, q\right)\right) .
$$

3.3. $G U(n, q)$ and its relatives. The paper $\mathrm{MR}$ proves that

$$
k(G U(n, q)) \leq q^{n} \prod_{i \geq 1} \frac{1+1 / q^{i}}{1-1 / q^{i}} \leq 8.26 q^{n} .
$$

Proposition 3.9 gives an asymptotic result.

Proposition 3.9. (1) For $q$ fixed, $\lim _{n \rightarrow \infty} \frac{k(G U(n, q))}{q^{n}}=\prod_{i \geq 1} \frac{1+1 / q^{i}}{1-1 / q^{i}}$.

(2) $q^{n}+q^{n-1} \leq k(G U(n, q)) \leq q^{n}+A q^{n-1}$ for a universal constant $A$; one can take $A=16$ for $q=2$ and $A=7$ for $q \geq 3$. Thus $\lim _{q \rightarrow \infty} \frac{k(G U(n, q))}{q^{n}}=1$, and the convergence is uniform in $n$.

Proof. Wall [W] shows that $k(G U(n, q))$ is the coefficient of $t^{n}$ in

$$
\prod_{i=1}^{\infty} \frac{1+t^{i}}{1-q t^{i}} .
$$


Thus $\frac{k(G U(n, q))}{q^{n}}$ is the coefficient of $t^{n}$ in

$$
\frac{1}{1-t} \prod_{i=1}^{\infty} \frac{1+t^{i} / q^{i}}{1-t^{i+1} / q^{i}}
$$

For the first assertion, use Lemma 3.2

For the second assertion, the lower bound comes from the easily proved fact (essentially on page 35 of [W]) that $G U(n, q)$ has $q^{n}+q^{n-1}$ many semisimple conjugacy classes. For the upper bound, the assertion when $q=2$ is immediate from the fact that $k(G U(n, q)) \leq 8.26 q^{n}$. For $q \geq 3$, recall that

$$
k(G U(n, q)) \leq q^{n} \prod_{i \geq 1} \frac{1+1 / q^{i}}{1-1 / q^{i}}
$$

Lemma 3.4 gives that

$$
\prod_{i \geq 1} \frac{1+1 / q^{i}}{1-1 / q^{i}}=\prod_{i \geq 1} \frac{1-1 / q^{2 i}}{\left(1-1 / q^{i}\right)^{2}} \leq \frac{1}{\left(1-1 / q-1 / q^{2}\right)^{2}} \leq 1+\frac{7}{q},
$$

where the last inequality is an easy calculus exercise.

Remarks. (1) The value of the limit in part (1) of Proposition 3.9 is 8.25... when $q=2$.

(2) As in the remark after Proposition 3.5. the convergence of $\frac{k(G U(n, q))}{q^{n}}$ to $\prod_{i \geq 1} \frac{1+1 / q^{i}}{1-1 / q^{i}}$ is $O\left(q^{-n(1 / 2-\epsilon)}\right)$ for any $0<\epsilon<1 / 2$. Indeed, subtracting off the simple pole at $t=1$ from the generating function in Proposition 3.9 gives that

$$
\frac{1}{1-t} \prod_{i=1}^{\infty} \frac{1+t^{i} / q^{i}}{1-t^{i+1} / q^{i}}-\frac{1}{1-t} \prod_{i \geq 1} \frac{1+1 / q^{i}}{1-1 / q^{i}}
$$

is analytic for all $|t|<q^{1 / 2}$, so the claim follows from Lemma 3.3 .

Macdonald [M1 derived useful formulas for $k(S U(n, q)), k(P G U(n, q))$ and $k(P S U(n, q))$. These involve the quantity

$$
\phi_{r}(n)=n^{r} \prod_{p \mid n}\left(1-p^{-r}\right)
$$

where the product is over all primes dividing $n$. He showed that

$$
\begin{aligned}
k(S U(n, q)) & =\frac{1}{q+1} \sum_{d \mid n, q+1} \phi_{2}(d) k(G U(n / d, q)), \\
k(P G U(n, q)) & =\frac{1}{q+1} \sum_{d \mid n, q+1} \phi_{1}(d) k(G U(n / d, q)),
\end{aligned}
$$

and

$$
k(P S U(n, q))=\frac{1}{(q+1) \operatorname{gcd}(n, q+1)} \sum_{d_{1}, d_{2}} \phi_{1}\left(d_{1}\right) \phi_{2}\left(d_{2}\right) k\left(G U\left(\frac{n}{d_{1} d_{2}}, q\right)\right),
$$

where the sum is over all pairs of divisors $d_{1}, d_{2}$ of $q+1$ such that $d_{1} d_{2}$ divides $n$. 
Proposition 3.10. $\quad$ (1) $q^{n-1} \leq k(S U(n, q)) \leq 8.26 q^{n-1}$.

(2) $k(S U(n, q)) \leq q^{n-1}+A q^{n-2}$ for a universal constant $A$; one can take $A=16$ for $q=2$ and $A=7$ for $q \geq 3$. Thus $\lim _{q \rightarrow \infty} \frac{k(S U(n, q))}{q^{n-1}}=1$, and the convergence is uniform in $n$.

(3) For $q$ fixed, $\lim _{n \rightarrow \infty} \frac{k(S U(n, q))}{q^{n-1}}=\frac{1}{(1+1 / q)} \prod_{i \geq 1} \frac{1+1 / q^{i}}{1-1 / q^{i}}$.

Proof. The lower bound in part (1) is immediate from Theorem 3.1. The upper bounds in parts (1) and (2) will be proved together. For $n \leq 7$ the upper bounds are checked directly from Macdonald's formula for $k(S U(n, q))$. Next suppose that $q=2$ and $n \geq 8$. Then Macdonald's formula for $k(S U(n, q))$ and the upper bound on $k(G U(n, q))$ give that

$$
k(S U(n, 2)) \leq \frac{8.26}{3}\left(2^{n}+8 \cdot 2^{n / 3}\right) \leq 8.26\left(2^{n-1}\right) .
$$

For $q \geq 3$, we claim that $k(S U(n, q)) \leq(1+7 / q) q^{n-1}$, which also implies the upper bound in part (1). Suppose that $n \geq 8$ is even (the case of odd $n$ is similar). Then Macdonald's formula and part (2) of Proposition 3.9 give that $k(S U(n, q))$ is at most

$$
\begin{aligned}
& (1+7 / q) \frac{\left(q^{n}+(q+1)^{2}\left(q^{n / 2}+\cdots+1\right)\right)}{q+1} \\
\leq & (1+7 / q)\left(q^{n-1}-q^{n-2}+q^{n-3} \cdots \pm 1+q^{n / 2+1}+2 q^{n / 2}+\cdots 2 q+1\right) \\
\leq & (1+7 / q) \cdot q^{n-1} .
\end{aligned}
$$

Part (3) follows from part (1) of Proposition 3.9 and Macdonald's formula for $k(S U(n, q))$ (argue as in the case of $S L$ ).

Corollary 3.11 gives bounds on $k(H)$ where $H$ is a group between $S U(n, q)$ and $G U(n, q)$ or between $P S U(n, q)$ and $P G U(n, q)$.

Corollary 3.11. (1) Suppose that $S U(n, q) \subseteq H \subseteq G U(n, q)$ and that $j$ is the index of $H$ in $G U(n, q)$. Then

$$
\frac{q^{n-1}(q+1)}{j} \leq k(H) \leq \frac{q+1}{j} k(S U(n, q)) \leq \frac{q^{n}+A q^{n-1}}{j},
$$

where $A$ is a universal constant. One can take $A=25$ for $q=2$ and $A=11$ for $q \geq 3$.

(2) Suppose that $P S U(n, q) \subseteq H \subseteq P G U(n, q)$ and that $j$ is the index of $H$ in $P G U(n, q)$. Then

$$
\frac{q^{n-1}}{j} \leq k(H) \leq \frac{\operatorname{gcd}(n, q+1)}{j} k(P S U(n, q)) \leq \frac{q^{n-1}+8 q^{n-2}}{j} .
$$

Proof. Let $H$ be as in part (1) of the corollary. Then $k(H) \geq \frac{k(G U(n, q))}{j} \geq \frac{q^{n-1}(q+1)}{j}$, where the first inequality is Lemma 2.1 and the second is the fact that $G U(n, q)$ has $q^{n-1}(q+1)$ semisimple conjugacy classes. Lemma 2.1 gives that $k(H) \leq$ $\frac{q+1}{j} k(S U(n, q))$. The inequality $(q+1) k(S U(n, q)) \leq q^{n}+A q^{n-1}$ with the stated $A$ values follows from part (2) of Proposition 3.10 .

Let $H$ be as in part (2) of the corollary. Then $k(H) \geq \frac{k(P G U(n, q))}{j} \geq \frac{q^{n-1}}{j}$, where the first inequality is Lemma 2.1 and the second is the fact that $P G U(n, q)$ has at least $q^{n-1}$ conjugacy classes (clear from Macdonald's formula and the fact 
that $G U(n, q)$ has $q^{n-1}(q+1)$ semisimple conjugacy classes). The inequality $k(H) \leq$ $\frac{\operatorname{gcd}(n, q+1)}{j} k(P S U(n, q))$ comes from Lemma 2.1. and the inequality $\operatorname{gcd}(n, q+1) k(P S U(n, q)) \leq q^{n-1}+8 q^{n-2}$ follows from Macdonald's formula for $k(P S U(n, q))$ and an analysis similar to that in Proposition 3.10 .

3.4. Symplectic groups. We next consider symplectic groups. We treat the cases $q$ odd and even separately.

Theorem 3.12. Let q be odd.

(1) $q^{n} \leq k(S p(2 n, q)) \leq q^{n} \prod_{i=1}^{\infty} \frac{\left(1+\frac{1}{q^{i}}\right)^{4}}{\left(1-\frac{1}{q^{i}}\right)} \leq 10.8 q^{n}$.

(2) $k(S p(2 n, q)) \leq q^{n}+A q^{n-1}$ for a universal constant $A$; one can take $A=30$ for $q=3$ and $A=12$ for $q \geq 5$. Thus

$$
\lim _{q \rightarrow \infty} \frac{k(S p(2 n, q))}{q^{n}}=1,
$$

and the convergence is uniform in $n$.

(3) For $q$ fixed, $\lim _{n \rightarrow \infty} \frac{k(S p(2 n, q))}{q^{n}}=\prod_{i=1}^{\infty} \frac{\left(1+\frac{1}{q^{i}}\right)^{4}}{\left(1-\frac{1}{q^{i}}\right)}$.

Proof. The lower bound in part (1) is immediate from Theorem 3.1

For $q$ odd, Wall [W] shows that $k(S p(2 n, q))$ is the coefficient of $t^{n}$ in the generating function

$$
\prod_{i=1}^{\infty} \frac{\left(1+t^{i}\right)^{4}}{1-q t^{i}}
$$

Rewrite this generating function as

$$
\prod_{i=1}^{\infty} \frac{1-t^{i}}{1-q t^{i}} \prod_{i=1}^{\infty} \frac{\left(1+t^{i}\right)^{4}}{1-t^{i}}
$$

Since all coefficients of powers of $t$ in the second infinite product are non-negative, it follows that

$$
k(S p(2 n, q)) \leq \sum_{m=0}^{n}\left(\text { Coef. } t^{n-m} \text { in } \prod_{i=1}^{\infty} \frac{1-t^{i}}{1-q t^{i}}\right)\left(\text { Coef. } t^{m} \text { in } \prod_{i=1}^{\infty} \frac{\left(1+t^{i}\right)^{4}}{1-t^{i}}\right) .
$$

Now $\prod_{i=1}^{\infty} \frac{1-t^{i}}{1-q t^{i}}$ is the generating function for the number of conjugacy classes in $G L(n, q)$. Hence the coefficient of $t^{n-m}$ in it is at most $q^{n-m}$. It follows that

$$
k(S p(2 n, q)) \leq q^{n} \sum_{m=0}^{n} \frac{1}{q^{m}}\left(\text { Coef. } t^{m} \text { in } \prod_{i=1}^{\infty} \frac{\left(1+t^{i}\right)^{4}}{1-t^{i}}\right) .
$$

Since the coefficients of $t^{m}$ in $\prod_{i=1}^{\infty} \frac{\left(1+t^{i}\right)^{4}}{1-t^{i}}$ are positive, it follows that

$$
\begin{aligned}
k(S p(2 n, q)) & \leq q^{n} \sum_{m=0}^{\infty} \frac{1}{q^{m}}\left(\text { Coef. } t^{m} \text { in } \prod_{i=1}^{\infty} \frac{\left(1+t^{i}\right)^{4}}{1-t^{i}}\right) \\
& =q^{n} \prod_{i=1}^{\infty} \frac{\left(1+\frac{1}{q^{i}}\right)^{4}}{\left(1-\frac{1}{q^{i}}\right)} .
\end{aligned}
$$


The term $\prod_{i=1}^{\infty} \frac{\left(1+\frac{1}{q^{i}}\right)^{4}}{\left(1-\frac{1}{q^{i}}\right)}$ is visibly maximized among odd prime powers $q$ when $q=3$.

Then it becomes $\frac{\prod_{i=1}^{\infty}\left(1-\frac{1}{9^{i}}\right)^{4}}{\prod_{i=1}^{\infty}\left(1-\frac{1}{3^{i}}\right)^{5}} \leq 10.8 q^{n}$ by Lemma 3.4 .

The upper bound in part (2) follows from the upper bound in part (1), Lemma 3.4. and basic calculus (argue as in the unitary case).

For part (3), note that $\frac{k(S p(2 n, q))}{q^{n}}$ is the coefficient of $t^{n}$ in

$$
\frac{1}{1-t} \prod_{i \geq 1} \frac{\left(1+t^{i} / q^{i}\right)^{4}}{\left(1-t^{i+1} / q^{i}\right)}
$$

Then use Lemma 3.2

Remark. The value of the limit in part (3) of Theorem 3.12 is $10.7 \ldots$ when $q=3$.

Next we treat the symplectic group in even characteristic.

Theorem 3.13. Let $q$ be even.

(1)

$$
1+\sum_{n \geq 1} k(S p(2 n, q)) t^{n}=\prod_{i=1}^{\infty} \frac{\left(1-t^{4 i}\right)}{\left(1-t^{4 i-2}\right)\left(1-t^{i}\right)\left(1-q t^{i}\right)} .
$$

(2) $q^{n} \leq k(S p(2 n, q)) \leq q^{n} \prod_{i=1}^{\infty} \frac{\left(1-1 / q^{4 i}\right)}{\left(1-1 / q^{4 i-2}\right)\left(1-1 / q^{i}\right)^{2}} \leq 15.2 q^{n}$.

(3) $k(S p(2 n, q)) \leq q^{n}+A q^{n-1}$ for a universal constant $A$; one can take $A=29$ for $q=2$ and $A=5$ for $q \geq 4$. Thus

$$
\lim _{q \rightarrow \infty} \frac{k(S p(2 n, q))}{q^{n}}=1,
$$

and the convergence is uniform in $n$.

(4) For $q$ fixed, $\lim _{n \rightarrow \infty} \frac{k(\operatorname{Sp}(2 n, q))}{q^{n}}=\prod_{i=1}^{\infty} \frac{\left(1-1 / q^{4 i}\right)}{\left(1-1 / q^{4 i-2}\right)\left(1-1 / q^{i}\right)^{2}}$.

Proof. Wall [W] showed that

$$
1+\sum_{n \geq 1} k(\operatorname{Sp}(2 n, q)) t^{n}=\frac{\chi(0,1, t)}{\prod_{i=1}^{\infty}\left(1-q t^{i}\right)},
$$

where $\chi$ is the $n \rightarrow \infty$ limit of a recursively defined function. Andrews [A2] proved the L-M-W conjecture that

$$
\chi(0,1, t)=\frac{\sum_{j=0}^{\infty} t^{j(j+1)}}{\prod_{i=1}^{\infty}\left(1-t^{i}\right)} .
$$

An identity of Gauss (page 23 of [A1]) states that

$$
\sum_{j=0}^{\infty} t^{j(j+1) / 2}=\prod_{i=1}^{\infty} \frac{1-t^{2 i}}{1-t^{2 i-1}}
$$

and the first assertion follows. 
For the second assertion, combining part (1) with the same trick as in the odd characteristic case gives that

$$
\begin{aligned}
k(S p(2 n, q)) & \leq q^{n} \prod_{i=1}^{\infty} \frac{\left(1-1 / q^{4 i}\right)}{\left(1-1 / q^{i}\right)^{2}\left(1-1 / q^{4 i-2}\right)} \\
& \leq q^{n} \prod_{i=1}^{\infty} \frac{\left(1-1 / 2^{4 i}\right)}{\left(1-1 / 2^{i}\right)^{2}\left(1-1 / 2^{4 i-2}\right)} \\
& \leq 15.2 q^{n} .
\end{aligned}
$$

The last step used Lemma 3.4. The lower bound in the second assertion is immediate from Theorem 3.1

The proofs of parts (3) and (4) are analogous to the proofs of parts (2) and (3) in the odd characteristic case.

Remark. The value of the limit in part (4) of Theorem 3.13 is $15.1 \ldots$ when $q=2$.

3.5. Orthogonal groups. This section gives the results for the orthogonal groups. We assume that the dimension of the underlying space is at least 3 (almost all of the results are valid for the two dimensional case as well, but the results are trivial in that case and the lower bounds do not always hold because the semisimple rank is 0 ).

First we treat the case of even dimension with $q$ odd.

Theorem 3.14. Let $q$ be odd.

(1) $\frac{q^{n}}{2} \leq k\left(O^{ \pm}(2 n, q)\right) \leq 9.5 q^{n}$.

(2) $k\left(O^{ \pm}(2 n, q)\right) \leq \frac{q^{n}}{2}+A q^{n-1}$ for a universal constant $A$; one can take $A=27$ for $q=3$ and $A=18$ for $q \geq 5$. Thus

$$
\lim _{q \rightarrow \infty} \frac{k\left(O^{ \pm}(2 n, q)\right)}{q^{n}}=\frac{1}{2}
$$

and the convergence is uniform in $n$.

(3) For fixed q,

$$
\begin{aligned}
& \lim _{n \rightarrow \infty} \frac{k\left(O^{ \pm}(2 n, q)\right)}{q^{n}} \\
= & \frac{1}{4 \prod_{i=1}^{\infty}\left(1-1 / q^{i}\right)}\left[\prod_{i=1}^{\infty}\left(1+1 / q^{i-1 / 2}\right)^{4}+\prod_{i=1}^{\infty}\left(1-1 / q^{i-1 / 2}\right)^{4}\right] .
\end{aligned}
$$

Proof. For the lower bound in part (1), Theorem 3.1 gives that $S O^{ \pm}(2 n, q)$ has at least $q^{n}$ semisimple classes, and at most two of these can fuse into one class in $O^{ \pm}(2 n, q)$. For the upper bound, clearly $k\left(O^{ \pm}(2 n, q)\right)$ is the sum/difference of $\frac{k\left(O^{+}(2 n, q)\right)+k\left(O^{-}(2 n, q)\right)}{2}$ and $\frac{k\left(O^{+}(2 n, q)\right)-k\left(O^{-}(2 n, q)\right)}{2}$. By upper bounding each of these terms, we will upper bound $k\left(O^{ \pm}(2 n, q)\right)$.

Wall [W] shows that $k\left(O^{+}(2 n, q)\right)+k\left(O^{-}(2 n, q)\right)$ is the coefficient of $t^{2 n}$ in the generating function

$$
\prod_{i=1}^{\infty} \frac{\left(1+t^{2 i-1}\right)^{4}}{1-q t^{2 i}} .
$$


Rewrite this generating function as

$$
\prod_{i=1}^{\infty} \frac{1-t^{2 i}}{1-q t^{2 i}} \prod_{i=1}^{\infty} \frac{\left(1+t^{2 i-1}\right)^{4}}{1-t^{2 i}}
$$

Arguing as in the proofs for the symplectic cases and using Lemma 3.4 the coefficient of $t^{2 n}$ is at most

$$
\begin{aligned}
& q^{n} \sum_{m \geq 0} \frac{1}{q^{m}}\left(\text { Coef. } t^{2 m} \text { in } \prod_{i \geq 1} \frac{\left(1+t^{2 i-1}\right)^{4}}{\left(1-t^{2 i}\right)}\right) \\
= & \frac{q^{n}}{2} \sum_{m \geq 0} \frac{1}{q^{m}}\left(\text { Coef. } t^{2 m} \text { in }\left[\prod_{i \geq 1} \frac{\left(1+t^{2 i-1}\right)^{4}}{\left(1-t^{2 i}\right)}+\prod_{i \geq 1} \frac{\left(1-t^{2 i-1}\right)^{4}}{\left(1-t^{2 i}\right)}\right]\right) \\
\leq & \frac{q^{n}}{2}\left[\prod_{i \geq 1} \frac{\left(1+t^{2 i-1}\right)^{4}}{\left(1-t^{2 i}\right)}+\prod_{i \geq 1} \frac{\left(1-t^{2 i-1}\right)^{4}}{\left(1-t^{2 i}\right)}\right]_{\mid t=3^{-.5}} \\
\leq & 16.3 q^{n} .
\end{aligned}
$$

Wall $\left[\mathrm{W}\right.$ ] shows that $k\left(O^{+}(2 n, q)\right)-k\left(O^{-}(2 n, q)\right)$ is the coefficient of $t^{n}$ in

$$
\prod_{i=1}^{\infty} \frac{\left(1-t^{2 i-1}\right)}{\left(1-q t^{2 i}\right)}
$$

Since this is analytic for $t<q^{-1}+\epsilon$, Lemmas 3.3 and 3.4 imply an upper bound of

$$
q^{n} \prod_{i=1}^{\infty} \frac{\left(1+1 / q^{2 i-1}\right)}{\left(1-1 / q^{2 i-1}\right)} \leq q^{n} \prod_{i=1}^{\infty} \frac{\left(1+1 / 3^{2 i-1}\right)}{\left(1-1 / 3^{2 i-1}\right)} \leq 2.4 q^{n} .
$$

Combining this with the previous paragraph gives that $k\left(O^{ \pm}(2 n, q)\right) \leq 9.5 q^{n}$.

For part (2), the $q=3$ case is immediate from part (1). For $q \geq 5$, the upper bound on $\frac{k\left(O^{+}(2 n, q)\right)+k\left(O^{-}(2 n, q)\right)}{q^{n}}$ in the proof of part (1) and the lower bound $k\left(O^{ \pm}(2 n, q)\right) \geq \frac{q^{n}}{2}$ yield that $k\left(O^{ \pm}(2 n, q)\right.$ is at most

$$
\frac{1}{2}\left[\prod_{i \geq 1} \frac{\left(1+1 / q^{i-1 / 2}\right)^{4}}{\left(1-1 / q^{i}\right)}+\prod_{i \geq 1} \frac{\left(1-1 / q^{i-1 / 2}\right)^{4}}{\left(1-1 / q^{i}\right)}\right]-\frac{1}{2} .
$$

The result follows from Lemma 3.4 (as in the unitary case) and basic calculus.

For the third assertion, $\frac{k\left(O^{+}(2 n, q)\right)+k\left(O^{-}(2 n, q)\right)}{q^{n}}$ is the coefficient of $t^{2 n}$ in

$$
\frac{1}{1-t^{2}} \prod_{i=1}^{\infty} \frac{\left(1+t^{2 i-1} / q^{i-1 / 2}\right)^{4}}{1-t^{2(i+1)} / q^{i}}
$$

By Lemma 3.2, as $n \rightarrow \infty$ this converges to

$$
\frac{1}{2 \prod_{i=1}^{\infty}\left(1-1 / q^{i}\right)}\left[\prod_{i=1}^{\infty}\left(1+1 / q^{i-1 / 2}\right)^{4}+\prod_{i=1}^{\infty}\left(1-1 / q^{i-1 / 2}\right)^{4}\right] .
$$


Recall that $k\left(O^{+}(2 n, q)\right)-k\left(O^{-}(2 n, q)\right)$ is the coefficient of $t^{n}$ in

$$
\prod_{i=1}^{\infty} \frac{\left(1-t^{2 i-1}\right)}{\left(1-q t^{2 i}\right)}
$$

Since this is analytic for $|t|<q^{-1 / 2}$, it follows from Lemma 3.3 that

$$
\lim _{n \rightarrow \infty} \frac{k\left(O^{+}(2 n, q)\right)-k\left(O^{-}(2 n, q)\right)}{q^{n}}=0 .
$$

Remark. The value of the limit in part (3) of Theorem 3.14 is $8.14 \ldots$ when $q=3$.

To treat even dimensional special orthogonal groups in odd characteristic, the following lemma will be helpful.

Lemma 3.15. Let $q$ be odd and let $G=S O^{ \pm}(n, q)$. Let $C=g^{G}$. Set $H=O^{ \pm}(n, q)$ containing $G$. The following are equivalent:

(1) $C=g^{H}$.

(2) $g$ leaves invariant an odd dimensional non-degenerate space $W$.

(3) Some Jordan block of $g$ corresponding to either the polynomial $z+1$ or the polynomial $z-1$ has odd size.

If all Jordan blocks of $g$ corresponding to both the polynomials $z \pm 1$ have even size, then $g^{H}$ is the union of two conjugacy classes of $G$.

Proof. Since $[H: G]=2$, the last statement follows from the equivalence of the first three conditions.

Suppose that $C=g^{H}$. It follows that $g$ centralizes some element $x \in H \backslash G$. Raising $x$ to an odd power, we may assume that the order of $x$ is a power of 2 and in particular that $x$ is semisimple. Since $\operatorname{det} x=-1$, it follows that the -1 eigenspace of $x$ is non-degenerate and odd dimensional. Thus (2) holds.

Conversely, assume (2). Taking $x=-1$ and $W$ on 1 on $W^{\perp}$ shows that $C_{H}(g)$ is not contained in $G$, whence (1) holds. Also, the subspace of $W$ corresponding to either the $z-1$ or $z+1$ space is odd dimensional, whence some Jordan block has odd size. Thus (2) implies (3).

Finally assume (3). By induction, we may assume that $g$ acts indecomposably (i.e. preserves no non-trivial orthogonal decomposition on the natural module). If $n$ is odd, then clearly (2) holds. So we may assume that $n$ is even. Replacing $g$ by $-g$ (if necessary), we may assume that $g$ is unipotent. By [LSe1, Theorem 2.12], it follows that either $g$ is a single Jordan block of odd size or has two Jordan blocks of even size. Since (3) holds, the latter case cannot hold. Thus, $g$ consists of a single Jordan block of odd size, whence (2) holds.

Theorem 3.16. Let $q$ be odd.

(1) $k\left(S O^{+}(2 n, q)\right)+k\left(S O^{-}(2 n, q)\right)$ is the coefficient of $t^{2 n}$ in

$$
\frac{3}{2} \prod_{i \geq 1} \frac{\left(1-t^{2 i}\right)^{2}}{\left(1-t^{4 i}\right)^{2}\left(1-q t^{2 i}\right)}+\frac{1}{2} \prod_{i \geq 1} \frac{\left(1+t^{2 i-1}\right)^{4}}{\left(1-q t^{2 i}\right)}
$$

(2) $k\left(S O^{+}(2 n, q)\right)-k\left(S O^{-}(2 n, q)\right)$ is the coefficient of $t^{n}$ in

$$
2 \prod_{i \geq 1} \frac{1}{\left(1+t^{i}\right)\left(1-q t^{2 i}\right)} .
$$

(3) $q^{n} \leq k\left(S O^{ \pm}(2 n, q)\right) \leq 7.5 q^{n}$. 
(4) $k\left(S O^{ \pm}(2 n, q)\right) \leq q^{n}+A q^{n-1}$ for a universal constant $A$; one can take $A=20$ for $q=3$ and $A=8$ for $q \geq 5$. Thus

$$
\lim _{q \rightarrow \infty} \frac{k\left(S O^{ \pm}(2 n, q)\right)}{q^{n}}=1
$$

and the convergence is uniform in $n$.

(5) For fixed q,

$$
\begin{aligned}
& \lim _{n \rightarrow \infty} \frac{k\left(S O^{ \pm}(2 n, q)\right)}{q^{n}} \\
= & {\left[\frac{3}{4} \prod_{i \geq 1} \frac{\left(1-1 / q^{i}\right)}{\left(1-1 / q^{2 i}\right)^{2}}+\frac{1}{8} \prod_{i \geq 1} \frac{\left(1+1 / q^{i-1 / 2}\right)^{4}}{\left(1-1 / q^{i}\right)}+\frac{1}{8} \prod_{i \geq 1} \frac{\left(1-1 / q^{i-1 / 2}\right)^{4}}{\left(1-1 / q^{i}\right)}\right] . }
\end{aligned}
$$

Proof. Clearly $k\left(S O^{+}(2 n, q)\right)+k\left(S O^{-}(2 n, q)\right)=2 A+B$, where $A$ is the sum over $O^{+}(2 n, q)$ and $O^{-}(2 n, q)$ of the number of classes which have determinant 1 and split into two $S O$ classes, and $B$ is the sum over $O^{+}(2 n, q)$ and $O^{-}(2 n, q)$ of the number of classes which have determinant 1 and do not split into two $S O$ classes. Applying Lemma 3.15] and arguing as on pages 41-42 of [W] gives that $A$ is the coefficient of $t^{2 n}$ in

$$
\prod_{i \geq 1} \frac{\left(1-t^{2 i}\right)^{2}}{\left(1-t^{4 i}\right)^{2}\left(1-q t^{2 i}\right)} .
$$

(The factor of $\left(1-t^{4 i}\right)^{-2}$ comes from the fact that the $z \pm 1$ partitions have only even parts which must occur with even multiplicity, and the other factor is precisely Wall's $\left.F_{0}^{+}(t)\right)$. To solve for $B$, note that $A+B$ is the sum over $O^{+}(2 n, q)$ and $O^{-}(2 n, q)$ of the number of classes which have determinant 1 . Such classes correspond to elements where the $z+1$ piece has even size, so arguing as on pages 41-42 of [W] (using his notation) gives that $A+B$ is the coefficient of $t^{2 n}$ in

$$
\begin{aligned}
& \frac{1}{2}\left[F_{+}^{+}(t)+F_{+}^{+}(-t)\right] F_{-}^{+}(t) F_{0}^{+}(t) \\
= & \frac{1}{2}\left[\prod_{i \geq 1} \frac{\left(1+t^{2 i-1}\right)^{4}}{\left(1-q t^{2 i}\right)}+\prod_{i \geq 1} \frac{\left(1-t^{2 i-1}\right)^{2}\left(1+t^{2 i-1}\right)^{2}}{\left(1-q t^{2 i}\right)}\right] .
\end{aligned}
$$

Calculating $2 A+B$ completes the proof of the first part of the theorem.

For the second assertion, apply Lemma 3.15 and argue as on pages 41-42 of [W] (using his notation) to conclude that the $O^{+}(2 n, q)$ number - the $O^{-}(2 n, q)$ number of conjugacy classes which have determinant 1 and split is the coefficient of $t^{2 n}$ in

$$
\prod_{i \geq 1} \frac{1}{\left(1-t^{4 i}\right)^{2}} F_{0}^{-}(t)=\prod_{i \geq 1} \frac{1}{\left(1+t^{2 i}\right)\left(1-q t^{4 i}\right)} .
$$

Again applying Lemma 3.15 and arguing as on pages 41-42 of [W], one sees that the $O^{+}(2 n, q)$ number - the $O^{-}(2 n, q)$ number of conjugacy classes which have determinant 1 and do not split is 0 . The second assertion follows.

The lower bound in part (3) is immediate from Theorem 3.1. For the upper bound, it follows from part (1) and elementary manipulations that $k\left(S O^{+}(2 n, q)\right)+$ 
$k\left(S O^{-}(2 n, q)\right)$ is the coefficient of $t^{2 n}$ in

$$
\left[\frac{3}{2} \prod_{i \geq 1} \frac{1-t^{4 i-2}}{1-t^{4 i}}+\frac{1}{2} \prod_{i \geq 1} \frac{\left(1+t^{2 i}\right)\left(1+t^{2 i-1}\right)^{4}}{\left(1-t^{4 i}\right)}\right] \prod_{i \geq 1} \frac{1-t^{2 i}}{1-q t^{2 i}}
$$

It is not difficult to see that the expression in square brackets in the previous equation has all coefficients non-negative when expanded as a power series in $t$ (use the fact that the coefficient of $t^{4 i-2}$ in $\left(1+t^{2 i-1}\right)^{4}$ is 6 ). Hence one can argue as in Theorem 3.14 to conclude that $k\left(S O^{+}(2 n, q)\right)+k\left(S O^{-}(2 n, q)\right)$ is at most $q^{n}$ multiplied by

$$
\left[\frac{3}{2} \prod_{i \geq 1} \frac{1-t^{4 i-2}}{1-t^{4 i}}+\frac{1}{4} \prod_{i \geq 1} \frac{\left(1+t^{2 i}\right)\left(1+t^{2 i-1}\right)^{4}}{\left(1-t^{4 i}\right)}+\frac{1}{4} \prod_{i \geq 1} \frac{\left(1+t^{2 i}\right)\left(1-t^{2 i-1}\right)^{4}}{\left(1-t^{4 i}\right)}\right]
$$

evaluated at $t=3^{-.5}$. This is at most $9.3 q^{n}$.

By part (2) and the fact that $2 \prod_{i \geq 1} \frac{1}{\left(1+t^{i}\right)\left(1-q t^{2 i}\right)}$ is analytic for $|t|<q^{-1}+\epsilon$, it follows from Lemma 3.3 that $k\left(S O^{+}(2 n, q)\right)-k\left(S O^{-}(2 n, q)\right)$ is at most

$$
2 q^{n} \prod_{i \geq 1} \frac{1}{\left(1-1 / q^{i}\right)\left(1-1 / q^{2 i-1}\right)} \leq 2 q^{n} \prod_{i \geq 1} \frac{1}{\left(1-1 / 3^{i}\right)\left(1-1 / 3^{2 i-1}\right)} \leq 5.6 q^{n} .
$$

This, together with the previous paragraph, completes the proof of the third assertion.

For part (4), the $q=3$ case is immediate from part (1). For $q \geq 5$, the proof of part (3) showed that $\frac{k\left(S O^{ \pm}(2 n, q)\right)}{q^{n}}$ is at most

$$
\begin{aligned}
& \frac{3}{2} \prod_{i \geq 1} \frac{1-1 / q^{2 i-1}}{1-1 / q^{2 i}}+\frac{1}{4} \prod_{i \geq 1} \frac{\left(1+1 / q^{i}\right)\left(1+1 / q^{i-1 / 2}\right)^{4}}{\left(1-1 / q^{2 i}\right)} \\
& +\frac{1}{4} \prod_{i \geq 1} \frac{\left(1+1 / q^{i}\right)\left(1-1 / q^{i-1 / 2}\right)^{4}}{\left(1-1 / q^{2 i}\right)}-1 .
\end{aligned}
$$

Using Lemma 3.4 (as in the unitary case), the result follows from basic calculus.

The proof of part (5) is nearly identical to the proof of part (3) in Theorem 3.14 .

Remark. The value of the limit in part (5) of Theorem 3.16 is $4.6 \ldots$ when $q=3$.

We next consider the odd dimensional case.

Theorem 3.17. Let $q$ be odd.

(1) $q^{n} \leq k(S O(2 n+1, q)) \leq q^{n} \prod_{i=1}^{\infty} \frac{\left(1-1 / q^{4 i}\right)^{2}}{\left(1-1 / q^{i}\right)^{3}\left(1-1 / q^{4 i-2}\right)^{2}} \leq 7.1 q^{n}$.

(2) $k(S O(2 n+1, q)) \leq q^{n}+A q^{n-1}$ for a universal constant $A$; one can take $A=19$ for $q=3$ and $A=8$ for $q \geq 5$. Thus

$$
\lim _{q \rightarrow \infty} \frac{k(S O(2 n+1, q))}{q^{n}}=1,
$$

and the convergence is uniform in $n$.

(3) For fixed $q, \lim _{n \rightarrow \infty} \frac{k(S O(2 n+1, q))}{q^{n}}=\prod_{i=1}^{\infty} \frac{\left(1-1 / q^{4 i}\right)^{2}}{\left(1-1 / q^{i}\right)^{3}\left(1-1 / q^{4 i-2}\right)^{2}}$.

(4) $k(O(2 n+1, q))=2 k(S O(2 n+1, q))$. 
Proof. Lusztig [Lus2] proves that $k(S O(2 n+1, q))$ is the coefficient of $t^{n}$ in the generating function

$$
\left(\sum_{j=0}^{\infty} t^{j(j+1)}\right)^{2} \prod_{i=1}^{\infty} \frac{1}{\left(1-t^{i}\right)^{2}\left(1-q t^{i}\right)} .
$$

By a result of Gauss (page 23 of [A1]), this is equal to

$$
\prod_{i=1}^{\infty} \frac{\left(1-t^{4 i}\right)^{2}}{\left(1-t^{4 i-2}\right)^{2}\left(1-t^{i}\right)^{2}\left(1-q t^{i}\right)}
$$

Using the same trick as in the unitary and symplectic cases one sees that

$$
k(S O(2 n+1, q)) \leq q^{n} \prod_{i=1}^{\infty} \frac{\left(1-1 / q^{4 i}\right)^{2}}{\left(1-1 / q^{i}\right)^{3}\left(1-1 / q^{4 i-2}\right)^{2}} .
$$

This is maximized for $q=3$ for which Lemma 3.4 yields an upper bound of $7.1 q^{n}$.

The lower bound follows by Steinberg's result on the number of semisimple classes - see Theorem 3.1.

The second part follows from part (1) (use Lemma 3.4 as in the unitary case and basic calculus), and the third part is proved using the same method used for the symplectic groups.

Since $O(2 n+1, q)=\mathbb{Z} / 2 \mathbb{Z} \times S O(2 n+1, q)$, the fourth result is clear.

Remark. The value of the limit in part (3) of Theorem 3.17 is $7.0 \ldots$ when $q=3$.

We now state similar results for the groups $\Omega^{ \pm}(n, q)$. The proofs of these results are somewhat long and are in [FG5].

Theorem 3.18 treats the even dimensional groups, while Theorem 3.19 treats the odd dimensional case.

It is convenient to define $\Omega^{*}(2 n, q)=\Omega^{+}(2 n, q)$ if $q \equiv 1 \bmod 4$ or $n$ is even and $\Omega^{-}(2 n, q)$ otherwise, and similarly for $S O$.

Note that for the even dimensional special orthogonal groups, one will be a direct product of its center and $\Omega$, and so the answer for $\Omega$ is precisely $1 / 2$ the answer for $S O$ (these are precisely the cases not mentioned in the next result).

Theorem 3.18. Let $q$ be odd.

(1) Set $j=2$ if $*=+$ and $j=1$ if $*=-$. Then $k\left(\Omega^{*}(2 n, q)\right)$ is the coefficient of $t^{2 n}$ in

$$
\begin{array}{r}
\frac{3}{8} \prod_{i \geq 1} \frac{1}{\left(1+t^{2 i}\right)^{2}\left(1-q t^{2 i}\right)}+\frac{1}{8} \prod_{i \geq 1} \frac{\left(1+t^{2 i-1}\right)^{4}}{\left(1-q t^{2 i}\right)} \\
+\frac{3}{2} \frac{\prod_{i \text { odd }}\left(1+t^{i}\right)^{2}}{\prod_{i \geq 1}\left(1-q t^{4 i}\right)}+j \frac{\prod_{i \text { odd }}\left(1-t^{2 i}\right)}{\prod_{i \geq 1}\left(1-q t^{4 i}\right)} .
\end{array}
$$

(2) For $n \geq 2, \frac{q^{n}}{2} \leq k\left(\Omega^{*}(2 n, q)\right) \leq 6.8 q^{n}$.

(3) $k\left(\Omega^{*}(2 n, q)\right) \leq \frac{q^{n}}{2}+A q^{n-1}$. One can take $A=16$ for $q=3$ and $A=8.5$ for $q \geq 5$. Thus

$$
\lim _{q \rightarrow \infty} \frac{k\left(\Omega^{+}(2 n, q)\right)}{q^{n}}=\frac{1}{2}
$$

and the convergence is uniform in $n$. 
(4) For fixed $q$,

$$
\begin{aligned}
& \lim _{n \rightarrow \infty} \frac{k\left(\Omega^{*}(2 n, q)\right)}{q^{n}}=\frac{1}{2} \cdot \lim _{n \rightarrow \infty} \frac{k\left(S O^{*}(2 n, q)\right)}{q^{n}} \\
= & \frac{3}{8} \prod_{i \geq 1} \frac{1}{\left(1+1 / q^{i}\right)^{2}\left(1-1 / q^{i}\right)}+\frac{1}{16} \prod_{i \geq 1} \frac{\left(1+1 / q^{i-1 / 2}\right)^{4}}{\left(1-1 / q^{i}\right)} \\
+ & \frac{1}{16} \prod_{i \geq 1} \frac{\left(1-1 / q^{i-1 / 2}\right)^{4}}{\left(1-1 / q^{i}\right)} .
\end{aligned}
$$

Remark. The value of the limits in part (4) of Theorem 3.18 is $2.3 \ldots$ when $q=3$.

The following result is for odd dimensional groups.

Theorem 3.19. Suppose that $q$ is odd.

(1) $k(\Omega(2 n+1, q))$ is the coefficient of $t^{2 n}$ in

$$
\frac{3}{4 t} \frac{\prod_{i \text { odd }}\left(1+t^{i}\right)^{2}}{\prod_{i \geq 1}\left(1-q t^{4 i}\right)}+\frac{1}{2} \prod_{i \geq 1} \frac{\left(1-t^{8 i}\right)^{2}}{\left(1-t^{8 i-4}\right)^{2}\left(1-t^{2 i}\right)^{2}\left(1-q t^{2 i}\right)} .
$$

(2) For $n \geq 2$, $\frac{q^{n}}{2} \leq k(\Omega(2 n+1, q)) \leq 7.3 q^{n}$.

(3) $k(\Omega(2 n+1, q)) \leq \frac{q^{n}}{2}+A q^{n-1}$. One can take $A=11$ for $q=3$ and $A=5.5$ for $q \geq 5$. Thus

$$
\lim _{q \rightarrow \infty} \frac{k(\Omega(2 n+1, q))}{q^{n}}=\frac{1}{2},
$$

and the convergence is uniform in $n$.

(4) For fixed q,

$$
\begin{aligned}
& \lim _{n \rightarrow \infty} \frac{k(\Omega(2 n+1, q))}{q^{n}}=\frac{1}{2} \cdot \lim _{n \rightarrow \infty} \frac{k(S O(2 n+1, q))}{q^{n}} \\
= & \frac{1}{2} \prod_{i \geq 1} \frac{\left(1-1 / q^{4 i}\right)^{2}}{\left(1-1 / q^{4 i-2}\right)^{2}\left(1-1 / q^{i}\right)^{3}} .
\end{aligned}
$$

In particular, we have:

Corollary 3.20. Fix an odd prime power $q$. Then

$$
\lim _{m \rightarrow \infty} \frac{k\left(\Omega^{ \pm}(m, q)\right)}{k\left(S O^{ \pm}(m, q)\right)}=\frac{1}{2} .
$$

We now turn to orthogonal groups in characteristic 2. Since the odd dimensional orthogonal groups are isomorphic to symplectic groups, we need only consider the even dimensional case. We let $S O^{ \pm}(2 n, q)$ denote the subgroup of index 2 in $O^{ \pm}(2 n, q)$ (corresponding to viewing $S O$ over the algebraic closure as the connected algebraic group with $O$ the disconnected group just as in any other characteristic). Since we are in characteristic $2, S O^{ \pm}(2 n, q)=\Omega^{ \pm}(2 n, q)$.

Theorem 3.21. Let $q$ be even.

(1)

$$
1+\sum_{n \geq 1} t^{n}\left[k\left(O^{+}(2 n, q)\right)+k\left(O^{-}(2 n, q)\right)\right]=\prod_{i=1}^{\infty} \frac{\left(1+t^{i}\right)\left(1+t^{2 i-1}\right)^{2}}{\left(1-q t^{i}\right)} .
$$


$(2)$

$$
\frac{q^{n}}{2} \leq k\left(O^{ \pm}(2 n, q)\right) \leq 15 q^{n}
$$

(3) $k\left(O^{ \pm}(2 n, q)\right) \leq \frac{q^{n}}{2}+A q^{n-1}$ for a universal constant $A$; one can take $A=29$ for $q=2$ and $A=9$ for $q \geq 4$. Thus

$$
\lim _{q \rightarrow \infty} \frac{k\left(O^{ \pm}(2 n, q)\right)}{q^{n}}=\frac{1}{2}
$$

and the convergence is uniform in $n$.

(4) For fixed $q, \lim _{n \rightarrow \infty} \frac{k\left(O^{ \pm}(2 n, q)\right)}{q^{n}}=\frac{1}{2} \prod_{i \geq 1} \frac{\left(1+1 / q^{i}\right)\left(1+1 / q^{2 i-1}\right)^{2}}{\left(1-1 / q^{i}\right)}$.

Proof. Combining [W] and [A2] shows that $k\left(O^{+}(2 n, q)\right)+k\left(O^{-}(2 n, q)\right)$ is the coefficient of $t^{n}$ in the generating function

$$
\frac{\sum_{j=-\infty}^{\infty} t^{j^{2}}}{\prod_{i=1}^{\infty}\left(1-t^{i}\right)\left(1-q t^{i}\right)} .
$$

The first assertion now follows from the special case of Jacobi's triple product identity (page 21 of [A1]):

$$
\sum_{n=-\infty}^{\infty} t^{n^{2}}=\prod_{i=1}^{\infty}\left(1-t^{2 i}\right)\left(1+t^{2 i-1}\right)^{2}
$$

Note that when the numerator of the generating function of part (1) is expanded as a series in $t$, all coefficients are positive. Arguing as for the unitary and symplectic groups gives that

$$
\begin{aligned}
k\left(O^{+}(2 n, q)\right)+k\left(O^{-}(2 n, q)\right) & \leq q^{n} \prod_{i \geq 1} \frac{\left(1+1 / q^{i}\right)\left(1+1 / q^{2 i-1}\right)^{2}}{\left(1-1 / q^{i}\right)} \\
& \leq q^{n} \prod_{i \geq 1} \frac{\left(1+1 / 2^{i}\right)\left(1+1 / 2^{2 i-1}\right)^{2}}{\left(1-1 / 2^{i}\right)} \\
& \leq 25.6 q^{n} .
\end{aligned}
$$

From [W], $k\left(O^{+}(2 n, q)\right)-k\left(O^{-}(2 n, q)\right)$ is the coefficient of $t^{n}$ in

$$
\prod_{i=1}^{\infty} \frac{1-t^{2 i-1}}{1-q t^{2 i}}
$$

Since this is analytic for $|t|<q^{-1}+\epsilon$, Lemma 3.3 gives that $k\left(O^{+}(2 n, q)\right)-$ $k\left(O^{-}(2 n, q)\right)$ is at most

$$
q^{n} \prod_{i=1}^{\infty} \frac{\left(1+1 / q^{2 i-1}\right)}{\left(1-1 / q^{2 i-1}\right)} \leq q^{n} \prod_{i=1}^{\infty} \frac{\left(1+1 / 2^{2 i-1}\right)}{\left(1-1 / 2^{2 i-1}\right)} \leq 4.2 q^{n} .
$$

Combining this with the the previous paragraph yields the upper bound in part (2).

For the lower bound in part $(2), S O^{ \pm}(2 n, q)$ is simply connected. Thus by Steinberg's theorem, the number of semisimple classes is exactly $q^{n}$ and so there are at least $q^{n} / 2$ in $O^{ \pm}(2 n, q)$ (because the index is 2 , at most two classes fuse into one).

The third and fourth parts are proved by the same method as in Theorem 3.14 
Remark. The value of the limit in part (4) of Theorem 3.21 is $12.7 \ldots$ when $q=2$.

Finally, we treat even characteristic special orthogonal groups. We let $S O^{ \pm}(2 n, q)$ $=\Omega^{ \pm}(2 n, q)$ denote the fixed points of the Frobenius map of the algebraic group (note that since $q$ is even, the algebraic group is both simply connected and of adjoint type). As usual, $O$ will denote the full isometry group of the form.

Theorem 3.22. Let $q$ be even.

(1)

$$
\begin{aligned}
& 2+\sum_{n \geq 1} t^{n}\left[k\left(S O^{+}(2 n, q)\right)+k\left(S O^{-}(2 n, q)\right)\right] \\
= & {\left[\frac{1}{2} \prod_{i \text { odd }} \frac{\left(1+t^{i}\right)^{2}}{\left(1-t^{i}\right)}+\frac{3}{2} \prod_{i=1}^{\infty} \frac{1}{\left(1+t^{i}\right)}\right] \prod_{i=1}^{\infty} \frac{1}{\left(1-q t^{i}\right)} . }
\end{aligned}
$$

$$
2+\sum_{n \geq 1} t^{n}\left[k\left(S O^{+}(2 n, q)\right)-k\left(S O^{-}(2 n, q)\right)\right]=2 \prod_{i=1}^{\infty} \frac{1}{\left(1+t^{i}\right)\left(1-q t^{2 i}\right)} .
$$

$$
q^{n} \leq k\left(S O^{ \pm}(2 n, q)\right) \leq 14 q^{n} .
$$

(4) $k\left(S O^{ \pm}(2 n, q)\right) \leq q^{n}+A q^{n-1}$ for a universal constant $A$; one can take $A=26$ for $q=2$ and $A=5$ for $q \geq 4$. Thus

$$
\lim _{q \rightarrow \infty} \frac{k\left(S O^{ \pm}(2 n, q)\right)}{q^{n}}=1,
$$

and the convergence is uniform in $n$.

(5) For fixed $q$, $\lim _{n \rightarrow \infty} \frac{k\left(S O^{ \pm}(2 n, q)\right)}{q^{n}}$ is equal to

$$
\frac{1}{4} \prod_{i \text { odd }} \frac{\left(1+1 / q^{i}\right)^{2}}{\left(1-1 / q^{i}\right)} \prod_{i=1}^{\infty} \frac{1}{\left(1-1 / q^{i}\right)}+\frac{3}{4} \prod_{i=1}^{\infty} \frac{1}{\left(1-1 / q^{2 i}\right)} .
$$

Proof. For part (1), it follows from [A2] and [Lus2] that if $k_{1}\left(S O^{ \pm}(2 n, q)\right)$ is the number of unipotent conjugacy classes of $S O^{ \pm}(2 n, q)$, then

$$
\begin{aligned}
& 1+\sum_{n \geq 1} t^{n}\left[k_{1}\left(S O^{+}(2 n, q)\right)+k_{1}\left(S O^{-}(2 n, q)\right)\right] \\
& =\frac{\frac{1}{2}+\sum_{n \geq 1} t^{n^{2}}}{\prod_{i \geq 1}\left(1-t^{i}\right)^{2}}+\frac{3}{2} \prod_{i \geq 1} \frac{1}{\left(1-t^{2 i}\right)}-1 .
\end{aligned}
$$

We claim that a conjugacy class of $O^{ \pm}(2 n, q)$ with an empty $z-1$ piece splits in $S O^{ \pm}(2 n, q)$, and that a conjugacy class of $O^{ \pm}(2 n, q)$ with a non-empty $z-1$ piece splits in $S O^{ \pm}(2 n, q)$ if and only if a unipotent element with that $z-1$ piece splits in the $S O$ (possibly of lower dimension) which contains it. Let $x \in O^{ \pm}(2 n, q)$. Write $V=V_{1} \perp V_{2}$, where $V_{1}$ is the kernel of $(x-1)^{2 n}$. Let $x_{i}$ denote the element of $O\left(V_{i}\right)$ that is the restriction of $x$ to $V_{i}$. Thus, the centralizer of $x$ is the direct product of the centralizers of $x_{i}$ in $O\left(V_{i}\right)$. Working over the algebraic closure we see that the centralizer of $x_{2}$ in $O\left(V_{2}\right)$ is isomorphic to the centralizer of some element of $G L(d)$ where $2 d=\operatorname{dim} V_{2}$. In particular, the centralizer of $x_{2}$ is connected and 
so is contained in $S O\left(V_{2}\right)$. Thus, if $V_{1}=0$, the class of $x$ splits. If $V_{1} \neq 0$, then the class of $x$ splits if and only if the class of $x_{1}$ splits in $O\left(V_{1}\right)$. This proves the claim.

Thus

$$
\begin{aligned}
& 2+\sum_{n \geq 1} t^{n}\left[k\left(S O^{+}(2 n, q)\right)+k\left(S O^{-}(2 n, q)\right)\right] \\
= & \left(2+\sum_{n \geq 1} t^{n}\left[k_{1}\left(S O^{+}(2 n, q)\right)+k_{1}\left(S O^{-}(2 n, q)\right)\right]\right) \prod_{i=1}^{\infty} \frac{\left(1-t^{i}\right)}{\left(1-q t^{i}\right)},
\end{aligned}
$$

where the term $\prod_{i \geq 1} \frac{\left(1-t^{i}\right)}{\left(1-q t^{i}\right)}$ is the even characteristic analog of $F_{0}^{+}(t)$ from page 41 of Wall [W] (and is derived the same way). Plugging in the generating function for $k_{1}^{ \pm}(S O(2 n, q))$ and using an identity of Gauss (page 21 of [A1]) that

$$
\frac{1}{2}+\sum_{n \geq 1} t^{n^{2}}=\frac{1}{2} \prod_{i \text { even }}\left(1-t^{i}\right) \prod_{i \text { odd }}\left(1+t^{i}\right)^{2},
$$

part (1) follows by elementary simplifications.

For part (2), arguing as in part (1) gives that

$$
\begin{aligned}
& 2+\sum_{n \geq 1} t^{n}\left[k\left(S O^{+}(2 n, q)\right)-k\left(S O^{-}(2 n, q)\right)\right] \\
= & \left(2+\sum_{n \geq 1} t^{n}\left[k_{1}\left(S O^{+}(2 n, q)\right)-k_{1}\left(S O^{-}(2 n, q)\right)\right]\right) \prod_{i=1}^{\infty} \frac{\left(1-t^{i}\right)}{\left(1-q t^{2 i}\right)},
\end{aligned}
$$

where the term $\prod_{i \geq 1} \frac{\left(1-t^{i}\right)}{\left(1-q t^{2 i}\right)}$ is the even characteristic analog of $F_{0}^{-}(t)$ from page 42 of Wall [W] (and is derived the same way). Page 153 of [Lus2] gives that

$$
2+\sum_{n \geq 1} t^{n}\left[k_{1}\left(S O^{+}(2 n, q)\right)-k_{1}\left(S O^{-}(2 n, q)\right)\right]=2 \prod_{i=1}^{\infty} \frac{1}{\left(1-t^{2 i}\right)},
$$

so part (2) follows.

The lower bound in part (3) is immediate from Theorem 3.1. For the upper bound, first note from part (1) that $k\left(S O^{+}(2 n, q)\right)+k\left(S O^{-}(2 n, q)\right)$ is the coefficient of $t^{n}$ in

$$
\left[\frac{1}{2} \prod_{i \text { odd }} \frac{\left(1+t^{i}\right)^{2}}{\left(1-t^{i}\right)} \prod_{i \geq 1} \frac{1}{\left(1-t^{i}\right)}+\frac{3}{2} \prod_{i \geq 1} \frac{1}{\left(1-t^{2 i}\right)}\right] \prod_{i=1}^{\infty} \frac{\left(1-t^{i}\right)}{\left(1-q t^{i}\right)} .
$$

Arguing as in Theorem 3.12 shows that $k\left(S O^{+}(2 n, q)\right)+k\left(S O^{-}(2 n, q)\right)$ is at most $q^{n}$ multiplied by

$$
\frac{1}{2} \prod_{i \text { odd }} \frac{\left(1+t^{i}\right)^{2}}{\left(1-t^{i}\right)} \prod_{i \geq 1} \frac{1}{\left(1-t^{i}\right)}+\frac{3}{2} \prod_{i \geq 1} \frac{1}{\left(1-t^{2 i}\right)}
$$

evaluated at $t=1 / q$. This is maximized at $q=2$, and is at most 15 . Using the fact that $k\left(S O^{ \pm}(2 n, q)\right) \geq q^{n}$, it follows that $k\left(S O^{ \pm}(2 n, q)\right) \leq 14 q^{n}$.

By part (2) and the fact that $2 \prod_{i \geq 1} \frac{1}{\left(1+t^{i}\right)\left(1-q t^{2 i}\right)}$ is analytic for $|t|<q^{-1}+\epsilon$, it follows from Lemma 3.3 that $k\left(S O^{+}(2 n, q)\right)-k\left(S O^{-}(2 n, q)\right)$ is at most

$$
2 q^{n} \prod_{i \geq 1} \frac{1}{\left(1-1 / q^{i}\right)\left(1-1 / q^{2 i-1}\right)} \leq 2 q^{n} \prod_{i \geq 1} \frac{1}{\left(1-1 / 2^{i}\right)\left(1-1 / 2^{2 i-1}\right)} \leq 17 q^{n} .
$$


This, together with the previous paragraph, completes the proof of the third assertion.

For part (4), the proof of part (3) yields that $\frac{k\left(S O^{ \pm}(2 n, q)\right)}{q^{n}}$ is at most

$$
\frac{1}{2} \prod_{i \text { odd }} \frac{\left(1+1 / q^{i}\right)^{2}}{\left(1-1 / q^{i}\right)} \prod_{i \geq 1} \frac{1}{\left(1-1 / q^{i}\right)}+\frac{3}{2} \prod_{i \geq 1} \frac{1}{\left(1-1 / q^{2 i}\right)}-1 .
$$

Using Lemma 3.4 (as in the unitary case), this upper bound is at most $2+\frac{A}{q}$ for a universal constant $A$. Since $k\left(S O^{ \pm}(2 n, q)\right) \geq q^{n}$ by part (3), the result follows. 3.14 .

The proof of part (5) is nearly identical to the proof of part (3) in Theorem

Remark. The limit in part (5) of Theorem 3.22 is $7.4 \ldots$ when $q=2$.

\section{TABLES OF CONJUGACY CLASS BOUNDS}

We tabulate some of the results in the previous section and summarize the corresponding results for exceptional groups. There are exact formulas for these class numbers and we refer the reader to $\mathrm{Lu} 2$. See also $\$ 8.18$ of $[\mathrm{Hu}$ and the references therein.

Table 1 contains the results for the exceptional groups. We give a polynomial upper bound for each type of exceptional group (this upper bound is valid for both the adjoint and simply connected forms of the group).

TABLe 1. Class numbers for exceptional groups

\begin{tabular}{|c||c|c|}
\hline$G$ & $k(G) \leq$ & Comments \\
\hline \hline${ }^{2} B_{2}(q)$ & $q+3$ & $q=2^{2 m+1}$ \\
\hline${ }^{2} G_{2}(q)$ & $q+8$ & $q=3^{2 m+1}$ \\
\hline$G_{2}(q)$ & $q^{2}+2 q+9$ & \\
\hline${ }^{2} F_{4}(q)$ & $q^{2}+4 q+17$ & $q=2^{2 m+1}$ \\
\hline${ }^{3} D_{4}(q)$ & $q^{4}+q^{3}+q^{2}+q+6$ & \\
\hline$F_{4}(q)$ & $q^{4}+2 q^{3}+7 q^{2}+15 q+31$ & \\
\hline$E_{6}(q)$ & $q^{6}+q^{5}+2 q^{4}+2 q^{3}+15 q^{2}+21 q+60$ & \\
\hline${ }^{2} E_{6}(q)$ & $q^{6}+q^{5}+2 q^{4}+4 q^{3}+18 q^{2}+26 q+62$ & \\
\hline$E_{7}(q)$ & $q^{7}+q^{6}+2 q^{5}+7 q^{4}+17 q^{3}+35 q^{2}+71 q+103$ & \\
\hline$E_{8}(q)$ & $q^{8}+q^{7}+2 q^{6}+3 q^{5}+10 q^{4}+16 q^{3}+40 q^{2}+67 q+112$ & \\
\hline
\end{tabular}

In particular, we see that $1 \leq k(G) / q^{r} \rightarrow 1$ as $q \rightarrow \infty$ in all cases in Table 1 . Also, $q^{r}<k(G) \leq q^{r}+14 q^{r-1}$ and $k(G) \leq 8 q^{r}$ in all cases in Table 1 .

Table 2 summarizes some of the results of the previous section on various (but not all) forms of the classical groups. These results all follow from the previous section.

In Table 3, we give bounds of the form $A q^{r}+B q^{r-1}$. We exclude $q=2$ or 3 in some cases - a similar bound follows from the previous table in those cases. 
TABLE 2. Class numbers for classical groups

\begin{tabular}{|c||c|c|}
\hline$G$ & $k(G) \leq$ & Comments \\
\hline \hline$S L(n, q)$ & $2.5 q^{n-1}$ & \\
\hline$S U(n, q)$ & $8.26 q^{n-1}$ & \\
\hline$S p(2 n, q)$ & $10.8 q^{n}$ & $q$ odd \\
\hline$S p(2 n, q)$ & $15.2 q^{n}$ & $q$ even \\
\hline$S O(2 n+1, q)$ & $7.1 q^{n}$ & $q$ odd \\
\hline$\Omega(2 n+1, q)$ & $7.3 q^{n}$ & $q$ odd \\
\hline$S O^{ \pm}(2 n, q)$ & $7.5 q^{n}$ & $q$ odd \\
\hline$\Omega^{ \pm}(2 n, q)$ & $6.8 q^{n}$ & $q$ odd \\
\hline$O^{ \pm}(2 n, q)$ & $9.5 q^{n}$ & $q$ odd \\
\hline$S O^{ \pm}(2 n, q)=\Omega^{ \pm}(2 n, q)$ & $14 q^{n}$ & $q$ even \\
\hline$O^{ \pm}(2 n, q)$ & $15 q^{n}$ & $q$ even \\
\hline
\end{tabular}

TABlE 3. Class numbers for classical groups II

\begin{tabular}{|c||c|c|}
\hline \multicolumn{1}{|c|}{$G$} & $k(G) \leq$ & Comments \\
\hline \hline$S L(n, q)$ & $q^{n-1}+3 q^{n-2}$ & \\
\hline$P G L(n, q)$ & $q^{n-1}+5 q^{n-2}$ & \\
\hline$S U(n, q)$ & $q^{n-1}+7 q^{n-2}$ & $q>2$ \\
\hline$P G U(n, q)$ & $q^{n-1}+8 q^{n-2}$ & $q>2$ \\
\hline$S p(2 n, q)$ & $q^{n}+12 q^{n-1}$ & $q>3$ odd \\
\hline$S p(2 n, q)$ & $q^{n}+5 q^{n-1}$ & $q>2$ even \\
\hline$S O(2 n+1, q)$ & $q^{n}+8 q^{n-1}$ & $q>3$ odd \\
\hline$\Omega(2 n+1, q)$ & $(1 / 2) q^{n}+5.5 q^{n-1}$ & $q>3$ odd \\
\hline$S O^{ \pm}(2 n, q)$ & $q^{n}+8 q^{n-1}$ & $q>3$ odd \\
\hline$\Omega^{ \pm}(2 n, q)$ & $(1 / 2) q^{n}+8.5 q^{n-1}$ & $q>3$ odd \\
\hline$O^{ \pm}(2 n, q)$ & $(1 / 2) q^{n}+18 q^{n-1}$ & $q>3$ odd \\
\hline$S O^{ \pm}(2 n, q)=\Omega^{ \pm}(2 n, q)$ & $q^{n}+5 q^{n-1}$ & $q>2$ even \\
\hline$O^{ \pm}(2 n, q)$ & $(1 / 2) q^{n}+9 q^{n-1}$ & $q>2$ even \\
\hline
\end{tabular}

\section{Conjugacy Classes in almost simple groups}

We use the results of the previous sections to obtains bounds on the class numbers for Chevalley groups. These bounds are close to best possible, but we improve these a bit in [FG5]. These bounds are used in [LOST to finish the proof of the Ore conjecture and are more than sufficient for our proof of the Boston-Shalev conjecture on derangements.

We first prove Theorem 1.1 . 
Proof. Since the number of semisimple classes in $G^{F}$ is at least $q^{r},(2)$ implies (3). Since any $F$-stable class intersects $G^{F}$, the number of $F$-stable classes is at most $k\left(G^{F}\right)$, and so (1) implies (4). Thus, it suffices to prove (1) and (2).

First assume that $G$ has type A. Apply Theorem [3.6, Proposition 3.10, and Corollaries 3.7 and 3.11 to conclude that (1) and (2) hold in this case.

If $G$ is exceptional, then the results follow by Table 1 in the previous section.

Now assume that $G$ has type B, C or D. Since the center has order at most 4 in all cases, to prove (1) it suffices to consider any form of the group (this may alter the constant, but only by a bounded amount, and in fact it changes only very little). Moreover, in characteristic 2, the adjoint and simply connected groups are the same, and so there is nothing to prove. So we may assume that the characteristic of the field is odd.

Suppose that $G$ has type B. The results have been proved for the group of adjoint type with constants 7.1 and 19 respectively. Suppose that $G$ is simply connected. Then $k(G) \leq 2 k(\Omega)$, whence the results hold by Theorem 3.19.

Next suppose that $G$ has type C. We have already proved the result for the simply connected group. So assume that $G$ is the adjoint form. Let $H=S p(2 r, q)$. Then $k(H / Z(H))<k(H) \leq 10.8 q^{r}$, whence $k(G) \leq 21.6 q^{r}$, and so (1) holds.

The number of semisimple classes in $H / Z(H)$ is at most $\left(q^{r}+t\right) / 2$, where $t$ is the number of $H$-semisimple classes invariant under multiplication by $Z(H)$. These correspond to monic polynomials of degree $r$ in $x^{2}$ with the set of roots invariant under inversion. The number of such is $q^{(r-1) / 2}$ if $r$ is odd and $q^{r / 2}$ if $r$ is even. Thus, $k(H / Z(H)) \leq(1 / 2) q^{r}+31 q^{r-1}$. Since $G / H$ has order 2 , this implies that $k(G) \leq q^{r}+62 q^{r-1}$, and so (2) holds.

Finally, consider the case that $G$ has type D (and we may assume that $r \geq 4$ ). Let $H=P \Omega^{ \pm}(2 r, q)$ be the simple group corresponding to $G$. Using Theorem 3.18 , we see that $k\left(P \Omega^{ \pm}(2 r, q)\right) \leq 6.8 q^{r}$, whence a straightforward argument shows that $k(G) \leq 4 k(H) \leq 27.2 q^{r}$.

Arguing as in the case of type $\mathrm{C}$, we see that $k\left(P \Omega^{ \pm}(2 r, q)\right) \leq(1 / 4) q^{r}+16 q^{r-1}+$ $q^{r / 2}$. Thus, $k(G) \leq 4 k\left(P \Omega^{ \pm}(2 r, q)\right) \leq q^{r}+68 q^{r-1}$.

Note that for simply connected groups or groups of adjoint type, we can do a bit better (and in particular for the simple groups). This follows by the proof above.

Corollary 5.1. Let $G$ be a simply connected simple algebraic group of rank $r$ over a field of positive characteristic. Let $F$ be a Steinberg-Lang endomorphism of $G$ with $G^{F}$ a finite Chevalley group over the field $\mathbb{F}_{q}$. Then

(1) $k\left(G^{F}\right) \leq 15.2 q^{r}$.

(2) $k\left(G^{F}\right) \leq q^{r}+40 q^{r-1}$.

Similarly, the proof of Theorem 1.1 also shows:

Corollary 5.2. Let $G$ be a simple algebraic group of adjoint type of rank $r$ over a field of positive characteristic. Let $F$ be a Steinberg-Lang endomorphism of $G$ with $G^{F}$ a finite Chevalley group over the field $\mathbb{F}_{q}$. Let $S$ be the socle of $G^{F}$ and assume that $S \leq H \leq G$. Then

(1) $k(H) \leq 27.2 q^{r}$.

(2) $k(H) \leq q^{r}+68 q^{r-1}$.

(3) The number of non-semisimple classes of $H$ is at most $68 q^{r-1}$.

Corollary 5.2 also leads to the following result which was used in GR. 
Proposition 5.3. Let $G$ be a finite almost simple group. Then $k(G) \leq|G|^{41}$.

Proof. For all cases other than the alternating and symmetric groups, this follows from Corollary 5.1 together with bounds on the size of the outer automorphism groups and a computer computation for small cases. For the symmetric groups, the result follows without difficulty from the two bounds $k\left(S_{n}\right) \leq \frac{\pi}{\sqrt{6(n-1)}} e^{\pi \sqrt{\frac{2 n}{3}}}$ ([VW], p. 140) and $n ! \geq(2 \pi)^{1 / 2} n^{n+1 / 2} e^{-n+1 /(12 n+1)}$ ([Fe, p. 52). By Corollary 2.7, $k\left(A_{n}\right) \leq k\left(S_{n}\right)$, and the two bounds also imply that $k\left(S_{n}\right) \leq\left(\frac{n !}{2}\right)^{.41}$ for $n \geq 6$. For $n=5, k\left(A_{5}\right)=5<(60)^{41}$ and $k\left(S_{5}\right)=7<(120)^{41}$.

The .41 gets much smaller as the rank grows (or as $n$ grows for $A_{n}$ ). Note that for $G=\mathrm{SL}\left(2,2^{a}\right), k(G)$ is very close to $|G|^{1 / 3}$.

We now consider general almost simple Chevalley groups $G$. Now we have to deal with all types of outer automorphisms. The proof we give below shows that in fact for large $q, k(G)$ is close to $q^{r} / e$, where $e=[\operatorname{Inndiag}(S): G \cap \operatorname{Inndiag}(S)]$.

We first need a lemma. See GLS for basic results about automorphisms of Chevalley groups.

Lemma 5.4. Let $S$ be a simple Chevalley group over the field of $q$ elements of rank $r \geq 2$. Let $x \in \mathrm{A} u t(S)$ with $x$ not an inner diagonal automorphism of $S$. Let $S \leq H \leq \operatorname{Inndiag}(S)$. Then the number of $x$ stable classes in $H$ is at most $D q^{r-1}$ for some universal constant $D$.

Proof. We may assume that $x$ has prime order $p$ modulo the group of inner diagonal automorphisms.

By Corollary 5.2 it suffices to consider semisimple classes of $S$ (since the number of non-semisimple classes is at most $A q^{r-1}$ for a universal constant $A$ ). If $x$ is in the coset of a Lang-Steinberg automorphism, then Shintani descent gives a much better bound. In any case, the stable classes will be in bijection with those in the centralizer and so there are at most $C q^{r / 2}$ invariant classes, whence the bound holds in this case.

The remaining cases are where $x$ induces a graph automorphism. We lift to the central cover $T$ of $S$ and let $H_{0}$ be the lift of $H$. It suffices to prove the result for $H_{0}$. By considering irreducible representations of $T$, we see that the number of stable semisimple classes in $T$ is $q^{r^{\prime}}$, where $r-1 \geq r^{\prime}$ is the number of orbits of $x$ on the Dynkin diagram of $S$. Similarly, for each $x$-invariant coset of $H_{0} / T$, there are at most $q^{r^{\prime}}$ invariant classes in each of those cosets. If $S$ is not of type A, there are at most 4 cosets. If $S$ is of type A, there are at most 2 invariant cosets. Thus, there are at most $4 q^{r-1}$ invariant semisimple classes.

We can now prove:

Theorem 5.5. Let $G$ be almost simple with socle $S$ that is a Chevalley group defined over the field of $q$ elements and has rank $r$. There is an absolute constant $D$ such that $k(G) \leq q^{r}+D(\log q) q^{r-1} \leq D^{\prime} q^{r}$.

Proof. Let $H$ be the subgroup of $G$ consisting of inner diagonal automorphisms. Let $X$ be the full group of inner diagonal automorphisms of $S$. Then $X=Y^{F}$, where $Y$ is the corresponding simple algebraic group of adjoint type, and so by Corollary 2.5, the number of semisimple classes in $H$ is precisely $[X: H]^{-1}$ times the number of semisimple classes in $X$. The number of non-semisimple classes in 
$H$ is at most $[X: H]$ times the number of non-semisimple classes of $X$. If $G$ is not of type A, then $[X: H] \leq 4$, and so by Theorem 1.1 $k(H) \leq q^{r}+E q^{r-1}$ for some universal constant $E$. If $G$ is of type A, we apply Corollaries 3.7 and 3.11 to conclude this as well.

First consider the case that $S=P S L(2, q)$. So $H=P S L(2, q)$ or $P G L(2, q)$. If $x \in G \backslash H$, then $x$ can be taken to be a field automorphism of order $e \geq 2$.

If $H=P G L(2, q)$, the number of stable semisimple classes is $q^{1 / e}+1$ and there is one (stable) unipotent class (the stable classes are precisely those of $P G L\left(2, q^{1 / e}\right)$ ). If $H=P S L(2, q)$, there are even fewer stable classes. Thus, the number of conjugacy classes in the coset $x H$ is at most $q^{1 / e}+2$, whence the result holds.

So we may assume that $r>1$.

Let $x \in G \backslash H$. By the previous result, the number of $x$-stable classes in $H$ is at most $E q^{r-1}$ for some universal constant $E$. Thus by Lemma 2.2, the number of conjugacy classes in $x H$ is at most $E q^{r-1}$. Since $[G: H] \leq 6 \log q$, it follows that

$$
k(G) \leq q^{r}+6 E(\log q) q^{r-1},
$$

and the result follows.

With a bit more effort, one can remove the $\log q$ factor in the previous result. Keeping track of the constants in the proof above gives Corollary 1.2 .

\section{Minimum Centralizer Sizes For the Finite Classical GRoups}

This section gives lower bounds on centralizer sizes in finite classical groups, and hence upper bounds on the size of the largest conjugacy class in a finite classical group. Formulas for the conjugacy classes sizes go back to Wall [W], but being quite complicated polynomials in $q$ effort is required to give explicit bounds. The bounds presented here hold for all values of $n$ and $q$ and are also applied in FG2] and [Sh].

The following standard notation about partitions will be used. Let $\lambda$ be a partition of some non-negative integer $|\lambda|$ into parts $\lambda_{1} \geq \lambda_{2} \geq \cdots$. Let $m_{i}(\lambda)$ be the number of parts of $\lambda$ of size $i$, and let $\lambda^{\prime}$ be the partition dual to $\lambda$ in the sense that $\lambda_{i}^{\prime}=m_{i}(\lambda)+m_{i+1}(\lambda)+\cdots$. It is also useful to define the diagram associated to $\lambda$ by placing $\lambda_{i}$ boxes in the $i$ th row. We use the convention that the row index $i$ increases as one goes downward. So the diagram of the partition (5441) is

and $\lambda_{i}^{\prime}$ can be interpreted as the size of the $i$ th column. The notation $(u)_{m}$ will denote $(1-u)(1-u / q) \cdots\left(1-u / q^{m-1}\right)$. This section freely uses Lemma 3.4 from Section 3 .

6.1. The general linear groups. The following result about partitions will be helpful.

Lemma 6.1. Let $\lambda$ be any partition. Then for $q \geq 2$,

$$
q^{\sum_{i}\left(\lambda_{i}^{\prime}\right)^{2}} \prod_{i}(1 / q)_{m_{i}(\lambda)} \geq q^{|\lambda|}(1-1 / q) .
$$


Proof. Define a function $f$ on partitions by $f(\lambda)=q^{\sum_{i}\left(\lambda_{i}^{\prime}\right)^{2}} \prod_{i}(1 / q)_{m_{i}(\lambda)}$. Let $\tau$ be a partition obtained from $\lambda$ by moving a box from a row of length $i$ to a row of length $j \geq i+1$. The idea is to show that $f(\lambda) \geq f(\tau)$. The result then follows because a sequence of such moves transforms any partition into the one-row partition, and $f$ evaluated on this partition is $q^{|\lambda|}(1-1 / q)$.

One checks that $\sum_{i}\left(\lambda_{i}^{\prime}\right)^{2}-\sum_{i}\left(\tau_{i}^{\prime}\right)^{2} \geq 2$, so if $i>1$, then

$$
\begin{aligned}
\frac{f(\tau)}{f(\lambda)} & \leq \frac{(1 / q)_{m_{i-1}(\lambda)+1}(1 / q)_{m_{i}(\lambda)-1}(1 / q)_{m_{j}(\lambda)-1}(1 / q)_{m_{j+1}(\lambda)+1}}{q^{2}(1 / q)_{m_{i-1}(\lambda)}(1 / q)_{m_{i}(\lambda)}(1 / q)_{m_{j}(\lambda)}(1 / q)_{m_{j+1}(\lambda)}} \\
& =\frac{\left(1-1 / q^{m_{i-1}(\lambda)+1}\right)\left(1-1 / q^{m_{j+1}(\lambda)+1}\right)}{q^{2}\left(1-1 / q^{m_{i}(\lambda)}\right)\left(1-1 / q^{m_{j}(\lambda)}\right)} \\
& \leq \frac{1}{q^{2}(1-1 / q)^{2}} \leq 1,
\end{aligned}
$$

as desired. For the $i=1$ case, the only difference in the above argument is that the term $\left(1-1 / q^{m_{i-1}(\lambda)+1}\right)$ does not appear.

Lemma 6.2 is well known and is proved by counting the non-zero elements in a degree $r$ extension of $\mathbb{F}_{q}$ by the degrees of their minimal polynomials.

Lemma 6.2. Let $N(q ; d)$ be the number of monic degree $d$ irreducible polynomials over the finite field $\mathbb{F}_{q}$, disregarding the polynomial $z$. Then

$$
\sum_{d \mid r} d N(q ; d)=q^{r}-1
$$

Lemma 6.3 will also be needed.

Lemma 6.3. For $s \geq 2$, $(1-1 / s)^{s} \geq e^{-(1+1 / s)}$.

Proof.

$$
\begin{aligned}
\log (1-1 / s)^{s} & =-1-\frac{1}{2 s}-\frac{1}{3 s^{2}}-\cdots \\
& \geq-1-\frac{1}{2 s}-\frac{1}{2 s^{2}}-\cdots \\
& =-1-\frac{1}{2 s(1-1 / s)} \\
& \geq-1-1 / s,
\end{aligned}
$$

where the final inequality uses that $s \geq 2$.

Theorem 6.4. The smallest centralizer size of an element of $G L(n, q)$ is at least $\frac{q^{n}(1-1 / q)}{e\left(1+\log _{q}(n+1)\right)}$.

Proof. It is well known that conjugacy classes of $G L(n, q)$ are parametrized by Jordan canonical form. That is, for each monic irreducible polynomial $\phi \neq z$, one picks a partition $\lambda(\phi)$ subject to the constraint $\sum_{\phi} \operatorname{deg}(\phi)|\lambda(\phi)|=n$. The corresponding centralizers sizes are well known (M2], page 181) and can be rewritten as

$$
\prod_{\phi} q^{\operatorname{deg}(\phi) \sum_{i}\left(\lambda(\phi)_{i}^{\prime}\right)^{2}} \prod_{i}\left(1 / q^{\operatorname{deg}(\phi)}\right)_{m_{i}(\lambda(\phi))}
$$

To minimize this expression, suppose that for each polynomial $\phi$ one knows the size $|\lambda(\phi)|$ of $\lambda(\phi)$. Lemma 6.1 shows that $\lambda(\phi)$ should be taken to be a one-row 
partition which would contribute $q^{|\lambda(\phi)| \cdot \operatorname{deg}(\phi)}\left(1-1 / q^{\operatorname{deg}(\phi)}\right)$. Letting $r$ be such that $\sum_{i=1}^{r} i N(q ; i) \geq n$, it follows that the minimal centralizer size is at least $q^{n} \prod_{i=1}^{r}\left(1-1 / q^{i}\right)^{N(q ; i)}$.

Observe that $r$ can be taken to be the smallest integer such that $q^{r}-1 \geq n$, because by Lemma 6.2

$$
\sum_{i=1}^{r} i N(q ; i) \geq \sum_{d \mid r} d N(q ; d)=q^{r}-1 .
$$

Since $N(q ; i) \leq q^{i} / i$, the minimum centralizer size is at least $q^{n} \prod_{i=1}^{r}\left(1-1 / q^{i}\right)^{q^{i} / i}$. Lemma 6.3 gives that the minimum centralizer size is at least $\frac{q^{n}(1-1 / q)}{e^{(1+1 / 2+\cdots+1 / r)}}$. To finish the proof use the bounds $1+1 / 2+\cdots+1 / r \leq 1+\log (r)$ and take $r=$ $1+\log _{q}(n+1)$.

Remark. Since the number of conjugacy classes of $G L(n, q)$ is less than $q^{n}$, one might hope that the largest conjugacy class size is at most $\frac{|G L(n, q)|}{c q^{n}}$, where $c$ is a constant. The proof of Theorem 6.4 shows this to be untrue. Indeed, the infinite product $\prod_{i}\left(1-1 / q^{i}\right)^{N(q ; i)}$ vanishes. This can be seen by setting $u=1 / q$ in the identity

$$
\prod_{i=1}^{\infty}\left(1-u^{i}\right)^{-N(q ; i)}=1+\frac{q-1}{q} \sum_{n \geq 1} u^{n} q^{n}
$$

(which holds since the coefficient of $u^{n}$ on both sides counts the number of monic degree $n$ polynomials with non-vanishing constant term).

6.2. The unitary groups. The method for the finite unitary groups is similar to that for $G L(n, q)$. As usual, we view $U(n, q)$ as a subgroup of $G L\left(n, q^{2}\right)$.

Lemma 6.5. Let $\lambda$ be any partition. Then for $q \geq 2$,

$$
q^{\sum_{i}\left(\lambda_{i}^{\prime}\right)^{2}} \prod_{i}(-1 / q)_{m_{i}(\lambda)} \geq q^{|\lambda|}(1+1 / q) .
$$

Proof. The argument is the same as for Lemma 6.1. Using the same notation as in that proof, except that now $f(\lambda)=q^{\sum_{i}\left(\lambda_{i}^{\prime}\right)^{2}} \prod_{i}(-1 / q)_{m_{i}(\lambda)}$, one obtains that

$$
\begin{aligned}
\frac{f(\tau)}{f(\lambda)} & \leq \frac{\left(1-(-1 / q)^{m_{i-1}(\lambda)+1}\right)\left(1-(-1 / q)^{m_{j+1}(\lambda)+1}\right)}{q^{2}\left(1-(-1 / q)^{m_{i}(\lambda)}\right)\left(1-(-1 / q)^{m_{j}(\lambda)}\right)} \\
& \leq \frac{(1+1 / q)^{2}}{q^{2}\left(1-1 / q^{2}\right)^{2}} \leq 1 .
\end{aligned}
$$

Given a polynomial $\phi$ with coefficients in $\mathbb{F}_{q^{2}}$ and a non-vanishing constant term, define a polynomial $\tilde{\phi}$ by

$$
\tilde{\phi}=\frac{z^{\operatorname{deg}(\phi)} \phi^{q}\left(\frac{1}{z}\right)}{[\phi(0)]^{q}}
$$

where $\phi^{q}$ raises each coefficient of $\phi$ to the $q$ th power. A polynomial $\phi$ is called self-conjugate if $\tilde{\phi}=\phi$, and an element in an extension field of $\mathbb{F}_{q^{2}}$ is called selfconjugate if its minimal polynomial over $\mathbb{F}_{q^{2}}$ is self-conjugate.

Lemma 6.6. Suppose that $r$ is odd. Then the number of non-zero non-self-conjugate elements in $\mathbb{F}_{q^{2 r}}$ viewed as an extension of $\mathbb{F}_{q^{2}}$ is $q^{2 r}-q^{r}-2$. 
Proof. Theorem 9 of [F2] shows that the number of self-conjugate elements of degree $i$ over $\mathbb{F}_{q^{2}}$ is 0 if $i$ is even and is $\sum_{d \mid i} \mu(d)\left(q^{i / d}+1\right)$ if $i$ is odd, where $\mu$ is the Moebius function. Thus Moebius inversion implies that the total number of self-conjugate elements of $\mathbb{F}_{q^{2 r}}$ is

$$
\sum_{i \mid r} \sum_{d \mid i} \mu(d)\left(q^{i / d}+1\right)=q^{r}+1,
$$

which implies the result.

Theorem 6.7. The smallest centralizer size of an element of $U(n, q)$ is at least $q^{n}\left(\frac{1-1 / q^{2}}{e\left(2+\log _{q}(n+1)\right)}\right)^{1 / 2}$.

Proof. For $n=1$ this is clear, so suppose that $n>1$. The conjugacy classes of $U(n, q)$ and their sizes were determined in $\mathrm{W}$. They are parametrized by the following analog of Jordan canonical form. For each monic irreducible polynomial $\phi \neq z$, one picks a partition $\lambda(\phi)$ subject to the two constraints that $\sum_{\phi} \operatorname{deg}(\phi)|\lambda(\phi)|=n$ and $\lambda(\phi)=\lambda(\tilde{\phi})$. The corresponding centralizer sizes are due to Wall and can be usefully rewritten as

$$
\begin{gathered}
\prod_{\phi \neq z, \phi=\tilde{\phi}} q^{\operatorname{deg}(\phi) \sum_{i}\left(\lambda(\phi)_{i}^{\prime}\right)^{2}} \prod_{i}\left(-1 / q^{\operatorname{deg}(\phi)}\right)_{m_{i}(\lambda(\phi))} \\
\cdot\left(\prod_{\{\phi, \tilde{\phi}\}, \phi \neq \tilde{\phi}} q^{\operatorname{deg}(\phi) \sum_{i}\left(\lambda(\phi)_{i}^{\prime}\right)^{2}} \prod_{i}\left(1 / q^{\operatorname{deg}(\phi)}\right)_{m_{i}(\lambda(\phi))}\right)_{q \mapsto q^{2}} .
\end{gathered}
$$

Here the $q \mapsto q^{2}$ means (in the second product over polynomials) to replace all occurrences of $q$ by $q^{2}$. Note that the second product is over unordered conjugate pairs of non-self-conjugate monic irreducible polynomials.

Note that the bound in Lemma 6.5 is greater than $q^{|\lambda|}$, whereas the bound in Lemma 6.1 is less than $q^{|\lambda|}$. Hence the minimum size centralizer will correspond to a conjugacy class whose characteristic polynomial has only non-selfconjugate irreducible polynomials as factors. Let $\tilde{M}(q ; i)$ denote the number of unordered pairs $\{\phi, \tilde{\phi}\}$, where $\phi$ is monic non-self-conjugate and irreducible of degree $i$ with coefficients in $F_{q^{2}}$. Then a lower bound for the smallest centralizer size is $q^{n} \prod_{i=1}^{r}\left(1-1 / q^{2 i}\right)^{\tilde{M}(q ; i)}$, where $r$ is such that $\sum_{i=1}^{r} 2 i \tilde{M}(q ; i) \geq n$. Take $r$ to be odd, and observe by Lemma 6.6 that

$$
\sum_{i=1}^{r} 2 i \tilde{M}(q ; i) \geq \sum_{i \mid r} 2 i \tilde{M}(q ; i)=q^{2 r}-q^{r}-2
$$

and that $q^{2 r}-q^{r}-2 \geq n$ if $q^{r} \geq n+1$ (since $n>1$ ). Since $\tilde{M}(q ; i) \leq \frac{q^{2 i}}{2 i}$, the smallest centralizer size is at least $q^{n} \prod_{i=1}^{r}\left(1-1 / q^{2 i}\right)^{\frac{q^{2 i}}{2 i}}$. Arguing as in the general linear case and applying Lemma 6.3 proves the theorem.

6.3. Symplectic and orthogonal groups. To begin the study of minimum centralizer sizes in symplectic and orthogonal groups, we treat the case of elements whose characteristic polynomial is $(z \pm 1)^{n}$. This will be done by two different methods. The first approach uses algebraic group techniques and gives the best bounds. The second approach is combinatorial but of interest, as it involves a new enumeration of unipotent elements in orthogonal groups. Note that there is no need 
to consider odd dimensional orthogonal groups in even characteristic, as these are isomorphic to symplectic groups.

Proposition 6.8. $\quad$ (1) The minimum centralizer size of an element in the group $\operatorname{Sp}(2 n, q)$ with characteristic polynomial $(z \pm 1)^{2 n}$ is at least $q^{n}$.

(2) In odd characteristic, the minimum centralizer size of an element with characteristic polynomial $(z \pm 1)^{2 n}$ in $O^{ \pm}(2 n, q)$ or $(z \pm 1)^{2 n+1}$ in $O(2 n+1, q)$ is at least $q^{n}$.

(3) In even characteristic, the minimum centralizer size of a unipotent element of $O^{ \pm}(2 n, q)$ is at least $2 q^{n-1}$.

Proof. We first work in the ambient algebraic group $G$ and connected component $H$. Let $g \in G(q)$. Let $B$ be a Borel subgroup of $H$ normalized by $g$ and $U$ its unipotent radical. First suppose that $g \in H$. It suffices to show that $C_{U}(g)$ has dimension at least $r$, the rank of $H$. Then the rational points in $C_{U}(g)$ have order a multiple of $q^{r}$, as required (cf. [FG1]). The centralizer in $B$ of a regular unipotent element in $B$ has dimension exactly $r$ in $B$. Since these elements are dense in $U$, the same is true for any such element.

Now suppose that $g$ is not in $H$. This only occurs in even characteristic with $G$ an orthogonal group. Now $G$ embeds in a symplectic group $L$ of the same dimension. Let $A$ be a maximal unipotent subgroup of $L$ containing $U$. Note that $g U$ contains regular unipotent elements of $L$, and so the subset of $g U$ consisting of regular unipotent elements is dense in $g U$.

We claim that $C_{U}(g)$ has dimension at least $r-1$. Once we have established that claim, it follows as above that the centralizer in $H(q)$ of $g$ is divisible by $q^{r-1}$, as required. Since $g$ is not in $H$, its centralizer in $G(q)$ has order at least twice as large. Since the regular unipotent elements in $g U$ are dense, it suffices to prove the claim for such an element.

Since $g$ is a regular unipotent element of $L$, it follows that $C_{L}(g)=C_{A}(g)$ has dimension $r$. On the other hand, we also see that the set of orthogonal groups containing $g$ is a one dimensional variety. (The orthogonal groups containing $g$ are in bijection with the $g$-invariant hyperplanes of the orthogonal module for $L$ that do not contain the $L$ fixed space. Since any such hyperplane must contain the image of $g-1$ which has codimension 2, we see the set of hyperplanes is a one dimensional variety.) Now $C_{U}(g)$ is precisely the stabilizer of the hyperplane corresponding to $H$ and so has codimension at most 1 in $C_{A}(g)$. Thus, $\operatorname{dim} C_{A}(g) \geq r-1$ (in fact, equality holds). This proves the claim and completes the proof.

We now give a more combinatorial approach to lower bounding centralizer sizes of elements whose characteristic polynomial is $(z \pm 1)^{n}$; this is complementary and yields different information. A crucial step in this approach is counting the number of unipotent elements in symplectic and orthogonal groups. Steinberg (see [C] for a proof) showed that if $G$ is a connected reductive group and $F: G \mapsto G$ is a Frobenius map, then the number of unipotent elements of $G^{F}$ is the square of the order of a $p$-Sylow, where $p$ is the characteristic. We remind the reader that

(1) $|S p(2 n, q)|=q^{n^{2}} \prod_{j=1}^{n}\left(q^{2 j}-1\right)$.

(2) $|O(2 n+1, q)|=2 q^{n^{2}} \prod_{j=1}^{n}\left(q^{2 j}-1\right)$ (in odd characteristic).

(3) $\left|O^{ \pm}(2 n, q)\right|=2 q^{n^{2}-n}\left(q^{n} \mp 1\right) \prod_{j=1}^{n-1}\left(q^{2 j}-1\right)$. 
This implies that the number of unipotent elements in $S p(2 n, q)$ is $q^{2 n^{2}}$. However the orthogonal groups are not connected, so Steinberg's theorem is not directly applicable. Nevertheless, in odd characteristic, unipotent elements always live in $\Omega$, so Steinberg's theorem does imply that the number of unipotent elements in $O(2 n+1, q)$ in odd characteristic is $q^{2 n^{2}}$ and that the number of unipotent elements of $O^{ \pm}(2 n, q)$ in odd characteristic is $q^{2\left(n^{2}-n\right)}$.

Proposition 6.11 uses generating functions to treat orthogonal groups in even characteristic. Along the way we obtain a formula for the number of unipotent elements (this turns out not to be a power of $q$ and seems challenging from the algebraic approach). Two combinatorial lemmas are needed.

Lemma 6.9 (Euler, A1]).

$$
\prod_{j=1}^{\infty}\left(\frac{1}{1-\frac{u}{q^{j}}}\right)=\sum_{n \geq 0} \frac{u^{n} q^{\left(\begin{array}{c}
n \\
2
\end{array}\right)}}{\left(q^{n}-1\right) \cdots(q-1)} .
$$

To state the second lemma, we require some notation (which will be used elsewhere in this subsection as well). Given a polynomial $\phi(z)$ with coefficients in $\mathbb{F}_{q}$ and non-vanishing constant term, define the "conjugate" polynomial $\phi^{*}$ by

$$
\phi^{*}=\frac{z^{\operatorname{deg}(\phi)} \phi\left(\frac{1}{z}\right)}{\phi(0)} .
$$

One calls $\phi$ self-conjugate if $\phi^{*}=\phi$. Note that the map $\phi \mapsto \phi^{*}$ is an involution. We let $N^{*}(q ; d)$ denote the number of monic irreducible self-conjugate polynomials of degree $d$ with coefficients in $\mathbb{F}_{q}$, and let $M^{*}(q ; d)$ denote the number of conjugate pairs of monic irreducible non-self-conjugate polynomials of degree $d$ with coefficients in $\mathbb{F}_{q}$.

Lemma 6.10 ([FNP $)$. Let $f=1$ if the characteristic is even and $f=2$ if the characteristic is odd. Then

$$
\begin{gathered}
\prod_{d \geq 1}\left(1-t^{d}\right)^{-N^{*}(q ; 2 d)}\left(1-t^{d}\right)^{-M^{*}(q ; d)}=\frac{(1-t)^{f}}{1-q t}, \\
\prod_{d \geq 1}\left(1+t^{d}\right)^{-N^{*}(q ; 2 d)}\left(1-t^{d}\right)^{-M^{*}(q ; d)}=1-t .
\end{gathered}
$$

Now we can enumerate unipotent elements in even characteristic orthogonal groups.

Proposition 6.11. Suppose that the characteristic is even. Then the number of unipotent elements of $O^{ \pm}(2 n, q)$ is $q^{2 n^{2}-2 n+1}\left(1+\frac{1}{q} \mp \frac{1}{q^{n}}\right)$.

Proof. Given a group $G$, we let $u(G)$ denote the proportion of elements of $G$ which are unipotent. We define generating functions $F^{ \pm}(t)$ by

$$
F^{ \pm}(t)=1+\sum_{n \geq 1} t^{n}\left(u\left(O^{+}(2 n, q)\right) \pm u\left(O^{-}(2 n, q)\right)\right) .
$$

There is a notion of cycle index for the orthogonal groups (see [F1] or [F2 for background), and the cycle indices for the sum and difference of the orthogonal groups factor. Setting all variables equal to 1 in the cycle index for the sum of 
$O^{+}(n, q)$ and $O^{-}(n, q)$, it follows that

$$
\frac{1+t}{1-t}=F^{+}(t) \frac{\prod_{j \geq 1}\left(1-\frac{t}{q^{2 j-1}}\right)}{1-t} .
$$

Let us make some comments about this equation. Here the $F^{+}(t)$ corresponds to the part of the cycle index for the polynomial $z-1$. The term $\frac{\prod_{j \geq 1}\left(1-\frac{t}{q^{2 j}-1}\right)}{1-t}$ corresponds to the remaining possible factors of the characteristic polynomial. This follows from the combinatorial identity

$$
\prod_{d \geq 1} \prod_{r \geq 1}\left(1+(-1)^{r} \frac{t^{d}}{q^{d r}}\right)^{-N^{*}(q ; 2 d)}\left(1-\frac{t^{d}}{q^{d r}}\right)^{-M^{*}(q ; d)}=\frac{\prod_{j \geq 1}\left(1-\frac{t}{q^{2 j-1}}\right)}{1-t},
$$

which is a consequence of Lemma 6.10 after reversing the order of the products. Solving for $F^{+}(t)$, one finds that

$$
F^{+}(t)=\frac{1+t}{\prod_{j \geq 1}\left(1-\frac{t}{q^{2 j-1}}\right)} .
$$

Taking the coefficient of $t^{n}$ and using Lemma 6.9, it follows that

$$
\begin{aligned}
& u\left(O^{+}(2 n, q)\right)+u\left(O^{-}(2 n, q)\right) \\
= & \frac{1}{q^{n-1}\left(1-1 / q^{2}\right) \cdots\left(1-1 / q^{2 n-2}\right)}\left(1+\frac{1}{q\left(1-1 / q^{2 n}\right)}\right) .
\end{aligned}
$$

Next we solve for $F^{-}(t)$. Setting all variables equal to 1 in the cycle index for the difference of $O^{+}(n, q)$ and $O^{-}(n, q)$, it follows that

$$
1=F^{-}(t) \prod_{j \text { even }}\left(1-\frac{t}{q^{j}}\right) .
$$

Here the $F^{-}(t)$ corresponds to the part of the cycle index for the polynomial $z-1$. The other term on the right hand side corresponds to the remaining possible factors of the characteristic polynomial. This follows from the combinatorial identity

$$
\prod_{d \geq 1} \prod_{r \geq 1}\left(1-(-1)^{r} \frac{t^{d}}{q^{d r}}\right)^{-N^{*}(q ; 2 d)}\left(1-\frac{t^{d}}{q^{d r}}\right)^{-M^{*}(q ; d)}=\prod_{j \text { even }}\left(1-\frac{t}{q^{j}}\right),
$$

which is a consequence of Lemma 6.10 after reversing the order of the products. Thus $F^{-}(t)=\prod_{j \text { even }}\left(1-\frac{t}{q^{j}}\right)^{-1}$. Taking the coefficient of $t^{n}$ and using Lemma 6.9, it follows that

$$
u\left(O^{+}(2 n, q)\right)-u\left(O^{-}(2 n, q)\right)=\frac{1}{q^{2 n}\left(1-1 / q^{2}\right) \cdots\left(1-1 / q^{2 n}\right)} .
$$

Having found formulas for $u\left(O^{+}(2 n, q)\right)+u\left(O^{-}(2 n, q)\right)$ and $u\left(O^{+}(2 n, q)\right)-$ $u\left(O^{-}(2 n, q)\right)$, one now solves for $u\left(O^{ \pm}(2 n, q)\right)$ giving the statement of the proposition.

Proposition 6.12 gives lower bounds on centralizer sizes for elements in symplectic and orthogonal groups whose characteristic polynomial is $(z \pm 1)^{n}$. Note that the bound of Proposition 6.8 was only slightly stronger.

Proposition 6.12. $\quad$ (1) The centralizer size of an element in the group $S p(2 n, q)$ whose characteristic polynomial has only factors $(z \pm 1)^{2 n}$ is at least $q^{n}\left(1-\frac{1}{q^{2}}-\frac{1}{q^{4}}\right)$. 
(2) In odd characteristic, the centralizer size of an element with characteristic polynomial $(z \pm 1)^{2 n+1}$ in $O(2 n+1, q)$ or $(z \pm 1)^{2 n}$ in $O^{ \pm}(2 n, q)$ is at least $q^{n}$

(3) In even characteristic, the centralizer size of a unipotent element in $O^{ \pm}(2 n, q)$ is at least $q^{n-1}\left(1-1 / q^{2}-1 / q^{4}\right)$.

Proof. For the first assertion, suppose without loss of generality that the element is unipotent. By Steinberg's theorem the total number of unipotent elements in $S p(2 n, q)$ is $q^{2 n^{2}}$. Hence the sum of the reciprocals of the centralizer sizes of unipotent elements is equal to $\frac{q^{2 n^{2}}}{|S p(2 n, q)|}$, from which it follows that the centralizer size of any unipotent element is at least

$$
\frac{|S p(2 n, q)|}{q^{2 n^{2}}}=q^{n}\left(1-1 / q^{2}\right) \cdots\left(1-1 / q^{2 n}\right) \geq q^{n}\left(1-1 / q^{2}-1 / q^{4}\right) .
$$

Note that the final inequality is Lemma 3.4

For the second assertion, suppose without loss of generality that the element is unipotent. By Steinberg's theorem the total number of unipotent elements in $O(2 n+1, q)$ is $q^{2 n^{2}}$. Thus the centralizer size of any unipotent conjugacy class of $O(2 n+1, q)$ is at least

$$
\frac{|O(2 n+1, q)|}{q^{2 n^{2}}}=2 q^{n}\left(1-1 / q^{2}\right) \cdots\left(1-1 / q^{2 n}\right) \geq q^{n},
$$

where the inequality uses Lemma 3.4 and the fact that $q \geq 3$. Similarly, in the even dimensional case with $q$ odd, the centralizer size is at least

$$
\frac{\left|O^{ \pm}(2 n, q)\right|}{q^{2\left(n^{2}-n\right)}}=2 q^{n}\left(1-1 / q^{2}\right) \cdots\left(1-1 / q^{2 n-2}\right)\left(1 \mp 1 / q^{n}\right) \geq q^{n} .
$$

In part (3) the characteristic is even, and using the count of unipotent elements in Proposition 6.11, it follows that the centralizer size of any unipotent element of $O^{ \pm}(2 n, q)$ is at least

$$
\begin{aligned}
& \frac{\left|O^{ \pm}(2 n, q)\right|}{q^{2 n^{2}-2 n+1}\left(1+\frac{1}{q} \mp \frac{1}{q^{n}}\right)} \\
& =\frac{2 q^{n-1}\left(1-1 / q^{2}\right) \cdots\left(1-1 / q^{2 n-2}\right)\left(1 \mp 1 / q^{n}\right)}{\left(1+1 / q \mp 1 / q^{n}\right)} \\
& \geq q^{n-1}\left(1-1 / q^{2}\right) \cdots\left(1-1 / q^{2 n-2}\right) \\
& \geq q^{n-1}\left(1-1 / q^{2}-1 / q^{4}\right) .
\end{aligned}
$$

Theorem 6.13 is the main result of this subsection. Note that there is no need to consider odd dimensional even characteristic orthogonal groups, as these are isomorphic to symplectic groups.

Theorem 6.13. (1) The centralizer size of an element of $S p(2 n, q)$ is at least

$$
q^{n}\left[\frac{1-1 / q}{2 e\left(\log _{q}(4 n)+4\right)}\right]^{1 / 2} .
$$

(2) The centralizer size of an element of $O^{ \pm}(2 n, q)$ is at least

$$
2 q^{n-1}\left[\frac{1-1 / q}{2 e\left(\log _{q}(4 n)+4\right)}\right]^{1 / 2} .
$$


(3) The centralizer size of an element of $S^{ \pm}(2 n, q)$ is at least

$$
q^{n}\left[\frac{1-1 / q}{2 e\left(\log _{q}(4 n)+4\right)}\right]^{1 / 2} .
$$

(4) Suppose that $q$ is odd. The centralizer size of an element of $O(2 n+1, q)$ is at least

$$
q^{n}\left[\frac{1-1 / q}{2 e\left(\log _{q}(4 n)+4\right)}\right]^{1 / 2} .
$$

Proof. First consider the case $S p(2 n, q)$. Wall W parametrized the conjugacy classes of $S p(2 n, q)$ and found their centralizer sizes. As in the general linear and unitary cases, the formula is multiplicative with terms coming from self-conjugate irreducible polynomials and also conjugate pairs of non-self-conjugate irreducible polynomials. By Proposition 6.8 a size $k$ partition corresponding to a polynomial $z-1$ or $z+1$ contributes at least a factor of $q^{k / 2}$. As with the unitary groups, one sees that a partition $\lambda$ from a self-conjugate irreducible polynomial $\phi$ contributes at least $q^{\operatorname{deg}(\phi) \cdot|\lambda| / 2}$ and that $\lambda$ associated with a pair $\{\phi, \bar{\phi}\}$ with $\phi$ monic non-selfconjugate irreducible contributes at least $q^{\operatorname{deg}(\phi) \cdot|\lambda|}\left(1-1 / q^{\operatorname{deg}(\phi)}\right)$. Then it follows that a lower bound for the smallest centralizer size is $q^{n} \prod_{i=1}^{2^{r}}\left(1-1 / q^{i}\right)^{M^{*}(q ; i)}$, where $2^{r}$ is chosen such that $\sum_{i=1}^{2^{r}} 2 i M^{*}(q ; i) \geq 2 n$. From [FNP], if $r \geq 1$, then

$$
\begin{aligned}
2^{r+1} M^{*}\left(q ; 2^{r}\right) & =2^{r} N\left(q ; 2^{r}\right)-2^{r} N^{*}\left(q ; 2^{r}\right) \\
& =\left(q^{2^{r}}-q^{2^{r-1}}\right)-2^{r} N^{*}\left(q ; 2^{r}\right) \geq q^{2^{r}}-2 q^{2^{r-1}} .
\end{aligned}
$$

Note that if $r \geq 2$, then $q^{2^{r}}-2 q^{2^{r-1}} \geq \frac{q^{2^{r}}}{2}$. It follows that if $r \geq 2$ and $q^{2^{r}} \geq 4 n$, then $\sum_{i=1}^{2^{r}} 2 i M^{*}(q ; i) \geq 2 n$. Thus we need a $2^{r}$ which is at least $\max \left\{4, \log _{q}(4 n)\right\}$, and one can find such a $2^{r}$ which is at most $2\left(\log _{q}(4 n)+4\right)$. Since $M^{*}(q ; i) \leq q^{i} / 2 i$, arguing as in the general linear case proves the first assertion of the theorem.

For the remaining assertions the contribution to the centralizer size coming from the part of the characteristic polynomial relatively prime to $z^{2}-1$ is the same for symplectic and orthogonal groups. Thus it is sufficient to focus on the part of the characteristic polynomial of the form $(z-1)^{a}(z+1)^{b}$, where $b=0$ if the characteristic is even. For $O^{ \pm}(2 n, q)$, the contribution must be at least $q^{\frac{a+b}{2}-1}$, for either the characteristic is odd and $a, b$ have the same parity and part (2) of Proposition 6.8 applies, or else the characteristic is even and part (3) of Proposition 6.8 applies. Note that if the element is not in $S O$, then the centralizer size is doubled in $O$, giving (3). If the element is in $S O$, then $a$ and $b$ are even. Thus, arguing as above, the minimum centralizer size is $q^{\frac{a+b}{2}}$ since $a$ and $b$ are both even, and (3) follows. For $O^{ \pm}(2 n+1, q)$, note by part (2) of Proposition 6.8 that the contribution must be at least $q^{\frac{a+b-1}{2}}$ since $a, b$ have unequal parity.

For exceptional groups (or more generally for groups of bounded rank), we have:

Lemma 6.14. Let $G$ be a connected simple exceptional algebraic group with $F$ a Frobenius endomorphism associated to the field of $q$ elements. If $g \in G^{F}$, then $\left|C_{G^{F}}(g)\right| \geq q^{r} / 26$.

Proof. Since $\operatorname{dim} C_{G}(g) \geq r$, it follows that $\left|C_{G^{F}}(g)\right| \geq(q-1)^{r}$. Since $r \leq 8$, the result follows for $q>2$. If $q=2$, the result follows by inspection (see [Lu2]). 
Note that Theorem 1.4 is an immediate consequence of the results in this section. In applications, we will have to deal with simple groups as well, so we state this. (In all cases except for type $\mathrm{A}$, the index of the simple group in the group of inner diagonal automorphisms is at most 4 . In type $A$, we use the results for $G L(n, q)$ or $U(n, q)$ and divide by $(q \mp 1) \operatorname{gcd}(q \mp 1, n)$ - one factor to pass to $S L$ or $S U$ and the other factor for the center of these groups.) Thus, we have:

Theorem 6.15. Let $S$ be a simple Chevalley group defined over the field of $q$ elements with $r$ the rank of the ambient algebraic group. There is a universal constant $A$ such that if $x$ is an inner diagonal automorphism of $S$, then

$$
\left|C_{S}(x)\right| \geq \frac{q^{r}}{A(\min \{q, r\})\left(1+\log _{q}(r)\right)} \geq \frac{q^{r-1}}{A\left(1+\log _{q}(r)\right)} .
$$

The result also applies to the full orthogonal group as well. The result fails for other graph automorphisms and field automorphisms.

\section{Conjugacy Classes of MAXIMAL SubGroups AND DERANGEMENTS}

We want to obtain bounds on the number of conjugacy classes of maximal subgroups of finite simple groups. We will then combine these results with our results on class numbers to obtain very strong results on the proportion of derangements in actions of simple and almost simple groups. We define $m(G)$ to be the number of conjugacy classes of maximal subgroups of $G$. Aschbacher and the second author AG conjectured that $m(G)<k(G)$ and proved this for $G$ solvable. Note that if $G$ is an elementary abelian 2-group, $m(G)+1=k(G)=|G|$.

A related conjecture (of Wall) is that the number of maximal subgroups of a finite group $G$ is less than $|G|$. Wall proved this for solvable groups. See [LPS] for more recent results.

First we note the following result, which we will not require - see [LS3] and combine this with [LMS].

Lemma 7.1. If $G=A_{n}$ or $S_{n}$, then $m(G) \leq n^{1+o(1)}$.

It follows by [LMS] that:

Theorem 7.2. Let $G$ be an almost simple Chevalley group of rank $r$ defined over the field of $q$ elements. Then $m(G) \leq c(r)+2 r \log \log q$.

The $\log \log q$ term comes from subfield groups.

This immediately gives a generalization of the Boston-Shalev conjecture in the case of bounded rank.

Theorem 7.3. Let $G$ be an almost simple group with socle $S$ a Chevalley group of fixed rank $r$ defined over $\mathbb{F}_{q}$. Assume that $G$ is contained in the group of innerdiagonal automorphisms of $S$. Let $\mathcal{M}(G)$ denote the set of maximal subgroups of $G$ that do not contain a maximal torus of $S$. Then

$$
\lim _{q \rightarrow \infty} \frac{\left|\bigcup_{M \in \mathcal{M}(G)} M\right|}{|G|}=0 .
$$

Proof. It follows by the basic results about maximal subgroups of $G$ (cf. [FG1]) and Corollary 1.2 that any maximal subgroup $M$ of $G$ either contains a maximal torus of $S$ or satisfies $k(M)<C q^{r-1}$ with $C$ a universal constant. Applying Theorem 
1.4 (with $r$ fixed) gives that for any maximal subgroup $M$ of $G$ not containing a maximal torus,

$$
\left|\bigcup_{g \in G} M^{g}\right| /|G| \leq k(M) \cdot \max _{x \in G} \frac{1}{\left|C_{G}(x)\right|}<O(1 / q)
$$

Thus, by Theorem 7.2 and the fact that $r$ is fixed,

$$
\frac{\left|\bigcup_{M \in \mathcal{M}(G)} M\right|}{|G|}<O\left(\frac{\log \log q}{q}\right),
$$

whence the result. Indeed, separating out the subfield case shows that $O(1 / q)$ is an upper bound in the equation above.

As in FG1, this gives:

Corollary 7.4. Let $G$ be an almost simple group with socle $S$ a Chevalley group of fixed rank $r$ defined over $\mathbb{F}_{q}$. Assume that $G$ is contained in the group of innerdiagonal automorphisms of $S$. Let $M$ be a maximal subgroup of $G$ not containing $S$ and set $\Omega=G / M$. Then there exists a universal constant $\delta>0$ such that $\delta(G, \Omega)>\delta$.

Remark. An inspection of the proof shows that the result holds for the proportion of derangements in a given coset of $S$. This is no longer true if we allow field automorphisms.

We now want to consider what happens for increasing $r$. In particular, it suffices to consider $r>8$ and so we restrict our attention to classical groups. The maximal subgroups have been classified by Aschbacher [As] and we consider the families individually.

We recall Aschbacher's theorem on maximal subgroups of classical Chevalley groups. We refer the reader to the description of the subgroups in As. See also KL.

Let $G$ be a classical Chevalley group with natural module $V$ of dimension $d$. Then a subgroup $H$ of $G$ falls into the following nine families. In particular, a maximal subgroup is either in $\mathcal{S}$ or is maximal in one of the families $\mathcal{C}_{i}$. We will write $\mathcal{C}_{i}(G)$ to denote the maximal subgroups of $G$ that are in the family $\mathcal{C}_{i}$. We let $\mathcal{S}(G)$ denote the maximal subgroups of $G$ in $\mathcal{S}$.

TABle 4. Aschbacher classes

\begin{tabular}{|l||c|}
\hline $\mathcal{C}_{1}$ & $H$ preserves either a totally singular or a non-degenerate subspace of $V$ \\
\hline $\mathcal{C}_{2}$ & $H$ preserves an additive decomposition of $V$ \\
\hline $\mathcal{C}_{3}$ & $H$ preserves an extension field structure of prime degree \\
\hline $\mathcal{C}_{4}$ & $V$ is tensor decomposable for $H$ \\
\hline $\mathcal{C}_{5}$ & $H$ is defined over a subfield of prime index \\
\hline $\mathcal{C}_{6}$ & $d$ is a power of a prime and $H$ normalizes a subgroup of symplectic type \\
\hline $\mathcal{C}_{7}$ & $V$ is tensor induced for $H$ \\
\hline $\mathcal{C}_{8}$ & $V$ is the natural module for a classical subgroup $H$ \\
\hline $\mathcal{S}$ & $H$ is the normalizer of an almost simple group $S$ and $H$ is not in $\mathcal{C}_{i}$ \\
\hline
\end{tabular}


It is easy to see (cf. GKS and [LPS, Lemmas 2.1, 2.4]):

Lemma 7.5. The number of conjugacy classes of maximal subgroups of $G$ in $\bigcup_{H \in \mathcal{C}_{i}}$ is at most $8 r \log r+r \log \log q$.

It is straightforward to see using Corollary 1.2 and the structure of the maximal subgroups in $\mathcal{C}_{i}$ that:

Lemma 7.6. Let $M \in \mathcal{C}_{i}(G)$ for $i>3$. There is a universal constant $C$ such that $k(M)<C q^{(r+1) / 2}$.

The only subgroups that are close to that bound are those in $\mathcal{C}_{8}$.

We will now prove:

Theorem 7.7. Let $G$ be a finite classical Chevalley group of rank $r$ over the field of $q$ elements. Let $X(G)$ denote the set of maximal subgroups of $G$ contained in $\mathcal{S}(G) \bigcup_{i=4}^{8} \mathcal{C}_{i}(G)$. For $r$ sufficiently large,

$$
\frac{\left|\bigcup_{H \in X(G)} H\right|}{|G|}<O\left(q^{-r / 3}\right) .
$$

We will prove this for each of the families, and taking unions implies the result. We remark that the theorem applies to any subgroup $G$ between the socle and the full isometry group of $V$. The result fails if we consider almost simple groups with field automorphisms allowed (see GMS for examples of so-called exceptional permutation groups and a classification of primitive almost simple exceptional permutation actions).

Of course, a trivial corollary is Theorem 1.3 . Note also that our estimate implies that the proportion of derangements in any coset of the simple group tends to 1 as well for the actions considered in Theorem 7.7 .

The idea of the proof is quite simple. Let $X(G)$ denote a set of subgroups of $G$ closed under conjugation. We want to show that the number of conjugacy classes of $G$ that intersect some element of $X(G)$ is at most $c(X)$. Then using our results for centralizer sizes (or equivalently an upper bound for sizes of conjugacy classes), we see that

$$
\left|\bigcup_{H \in X(G)} H\right| \leq \frac{c(X) A|G|\left(1+\log _{q} r\right)}{q^{r-1}}
$$

or

$$
\frac{\left|\bigcup_{H \in X(G)} H\right|}{|G|} \leq \frac{c(X) A\left(1+\log _{q} r\right)}{q^{r-1}}
$$

where $A$ is a universal constant. So we only need show that $c(X)$ is at most $O\left(q^{(2 / 3) r-2}\right)$ in each case.

We note that for a fixed simple group $S$, the number of embeddings of $S$ into $G$ is certainly bounded from above by $2 r k(\hat{S})$, where $\hat{S}$ is the universal cover of $S$ (note that $k(\hat{S})$ is an upper bound for the number of representations and the factor $2 r$ comes from the fact that we may have representations which are inequivalent in the simple classical group but become conjugate in the full group of isometries). The arguments vary slightly depending upon the family we are considering, but the basic idea is the same in all cases. 
Lemma 7.8. Let $G$ be a finite classical Chevalley group of rank $r$ over the field of $q$ elements. Let $X(G)$ denote the set of maximal subgroups of $G$ contained in $\mathcal{C}_{i}(G)$ for $i>3$. Then for $r$ sufficiently large,

$$
\frac{\left|\bigcup_{H \in X(G)} H\right|}{|G|}<O\left(q^{-r / 3}\right) .
$$

Proof. By Lemmas 7.5 and 7.6. one knows that $\bigcup_{H \in X(G)} H$ is the union of at most $C q^{(r+1) / 2}(8 r \log r+r \log \log q)$ conjugacy classes of $G$. By Theorem 6.15, each class has size at most $A|G|\left(1+\log _{q}(r)\right) / q^{r-1}$, with $A$ a universal constant, whence the result.

We now consider $\mathcal{S}(G)$. It is convenient to split $\mathcal{S}(G)$ into 4 subclasses defined as follows (we keep the notation as above). First recall that if $S$ is a quasisimple Chevalley group in characteristic $p$ and $V$ is an absolutely irreducible module, then $V=V(\lambda)$ for some dominant weight $\lambda$ (in particular, the representation extends to the algebraic group). Write $\lambda=\sum a_{i} \lambda_{i}$ with the $a_{i}$ non-negative integers and the $\lambda_{i}$ are the fundamental weights. A restricted representation is one with $a_{i}<p$ for all $i$. By the Steinberg tensor product theorem, every module is a tensor product of Frobenius twists of restricted modules (over the algebraic closure). See [J, St2] for details of this theory.

$\mathcal{S}_{1} S$ is alternating or sporadic;

$\mathcal{S}_{2} S$ is a Chevalley group in characteristic not dividing $q$;

$\mathcal{S}_{3} S$ is a Chevalley group in characteristic dividing $q$ and the representation is not restricted; and

$\mathcal{S}_{4} S$ is a Chevalley group in characteristic dividing $q$, and the representation is restricted.

Lemma 7.9. For $r$ sufficiently large, we have:

(1) The number of conjugacy classes of maximal subgroups in $\mathcal{S}_{1}(G)$ is at most $O\left(r^{1 / 2} e^{10 r^{1 / 4}}\right) ;$ and

$$
\frac{\left|\bigcup_{M \in \mathcal{S}_{1}(G)} M\right|}{|G|}<O\left(q^{-r / 3}\right) .
$$

Proof. We may assume that $r$ is sufficiently large such that there are no sporadic groups in $\mathcal{S}_{1}(G)$ or alternating groups of degree less than 17 .

Let $d$ be the dimension of the natural module for our classical group. So $d \leq$ $2 r+1$.

It follows by [GT1, Lemma 6] that either the module is the natural permutation module for the symmetric group or the dimension $d$ of the module satisfies $d \geq$ $\left(m^{2}-5 m+2\right) / 2$. Since $d \leq 2 r+1$, this implies that, for $r$ sufficiently large, aside from the natural permutation module, $m \leq 3 r^{1 / 2}$.

By the comments preceding Lemma 7.8, the number of embeddings of $A_{m}$ into $G$ is at most $2 r k\left(\widehat{A_{m}}\right) \leq 4 r k\left(A_{m}\right)$. Thus the number of conjugacy classes of elements of $\mathcal{S}_{1}(G)$ is at most

$$
(4 r) \sum_{m=5}^{3 r^{1 / 2}} 2 k\left(A_{m}\right) \leq 12 r^{3 / 2} k\left(A_{\left\lfloor 3 r^{1 / 2}\right\rfloor}\right) .
$$


Recalling from Corollary 2.7 that $k\left(A_{m}\right) \leq k\left(S_{m}\right)$, and using the bound $k\left(S_{m}\right) \leq$ $C m^{-1} \exp \left[\pi(2 m / 3)^{1 / 2}\right]$ which follows from the known asymptotic behavior of the partition function (A1, p. 70), one concludes that the number of conjugacy classes of elements of $\mathcal{S}_{1}(G)$ is at most $O\left(r e^{5 r^{1 / 4}}\right)$.

Excluding the natural representations, the number of conjugacy classes of each $M \in \mathcal{S}_{1}(G)$ is at most $2 k\left(A_{\left\lfloor 3 r^{1 / 2}\right\rfloor}\right) \leq O\left(r^{-1 / 2} e^{5 r^{1 / 4}}\right)$. Thus, excluding the embedding of $A_{m}$ into $G$ via the natural module, the total number of conjugacy classes of $G$ in the union of maximal subgroups in $\mathcal{S}_{1}(G)$ is at most $O\left(r^{1 / 2} e^{10 r^{1 / 4}}\right)$.

Finally, consider the natural embedding of $A_{m}$ or $S_{m}$ into $G$. Then $m=d+1$ or $d+2$ (depending upon the characteristic). Moreover, since the representation is self-dual, $G$ is either symplectic or orthogonal. Thus, there are at most 8 conjugacy classes of such maximal subgroups, each with at most $k\left(S_{2 r+3}\right)$ classes. Thus, these maximal subgroups contribute at most $O\left(r^{-1} \exp \left[\pi(4 r / 3)^{1 / 2}\right]\right)$ conjugacy classes of $G$.

Hence the total number of conjugacy classes of $G$ represented in $\bigcup_{M \in \mathcal{S}_{1}(G)}$ is at most $O\left(r^{-1} \exp \left(4 r^{1 / 2}\right)\right)$. Using Theorem 6.15 which gives an upper bound for the size of a conjugacy class, gives the result.

Lemma 7.10. For $r$ sufficiently large, we have:

(1) The number of conjugacy classes of maximal subgroups in $\mathcal{S}_{2}(G)$ is at most $O\left(r^{3}\right) ;$ and

$$
\frac{\left|\bigcup_{M \in \mathcal{S}_{2}(G)} M\right|}{|G|}<O\left(r^{4}\left(1+\log _{q}(r)\right) / q^{r-1}\right) .
$$

Proof. For part (1) we argue very much as in [LPS. Let $S$ be the socle. By the results of various authors on minimal dimensions of projective representations (see [T, Table 2]) and Corollary 1.2, it follows that there is a universal positive constant $A$ such that:

(a) $k(M) \leq A r$ for any $M \in \mathcal{S}_{2}(G)$,

(b) there are at most $A r$ possibilities for $S$ up to isomorphism, and

(c) $k(\hat{S}) \leq A r$ for each possible $S$.

By (b) and (c), the number of conjugacy classes of maximal subgroups of $G$ in $\mathcal{S}_{2}(G)$ is at most $O\left(r^{3}\right)$ (the extra $r$ comes from the possibility of equivalent representations which are not conjugate in $G$ ), and so (1) holds.

By (a) and (1), the number of conjugacy classes in the union of all maximal subgroups in $\mathcal{S}_{2}(G)$ is at most $O\left(r^{4}\right)$. Now (2) follows by this and Theorem6.15.

Lemma 7.11. For $r$ sufficiently large, we have:

(1) The number of conjugacy classes of maximal subgroups in $\mathcal{S}_{3}(G)$ is at most $O(2 r \log r) ;$ and

$$
\frac{\left|\bigcup_{M \in \mathcal{S}_{3}(G)} M\right|}{|G|}<O\left(q^{-r / 3}\right) .
$$

Proof. Since the representation is not restricted and $M$ is maximal, it follows by Steinberg's tensor product theorem that the representation must be the tensor product of Frobenius twists of some restricted representation. By [GT2, Lemma 26], $N_{G}(M)$ also preserves this tensor product (over the algebraic closure). 
Since $M$ is maximal, this implies that $M$ is a classical group over a larger field and that $V$ is the tensor product of Frobenius twists of the natural module for the classical group (and so this module is defined over the smaller field). Thus, there will be at most $2 r \log r$ choices for the class of $M$ (essentially depending upon writing the dimension as a power of a positive integer).

Indeed, let $d$ be the dimension of the natural module. Write $d=m^{e}$ with $e>1$. Then the socle of $M$ is a classical group over the field of size $q^{e}$ and of rank less than $m$. Thus, by Corollary 1.2, $k(M) \leq O\left(q^{e m}\right) \leq O\left(q^{3 r^{1 / 2}}\right)$.

It follows that $\bigcup_{M \in \mathcal{S}_{3}(G)} M$ contains at most $O\left(r \log r q^{3 r^{1 / 2}}\right)$ conjugacy classes of $G$. Now apply Theorem 6.15 to conclude that (2) holds.

Lemma 7.12. For $r$ sufficiently large with $p$ the characteristic of $G$, we have:

(1) The number of conjugacy classes of maximal subgroups in $\mathcal{S}_{4}(G)$ is at most $O\left(r^{3 / 2} p^{3 r^{1 / 2}}\right) ;$ and

$$
\frac{\left|\bigcup_{M \in \mathcal{S}_{4}(G)} M\right|}{|G|}<O\left(q^{-r / 2}\right) .
$$

Proof. The restricted representations of the groups of Ree type (i.e. one of ${ }^{2} B_{2},{ }^{2} G_{2},{ }^{2} F_{4}$ ) have bounded dimension, and so we may ignore these.

If $S$ is an untwisted Chevalley group over the field of $s$ elements, then every restricted representation is defined over that field and so $s$ must be the field size for the natural module for $G$. If $S$ is twisted, then any representation is defined over the field of $s$ elements or $s^{d}$ elements where $d \leq 3$ (depending upon the twist).

It also follows by Lu1 that the rank of $S$ is at most $3 r^{1 / 2}$.

Thus there are at most $D r^{1 / 2}$ choices for $S$ for an absolute constant $D$, and the number of possible representations is at most $p^{3 r^{1 / 2}}$. Thus, the number of possible conjugacy classes is at most $O\left(r^{3 / 2} p^{3 r^{1 / 2}}\right)$; the extra $r$ comes from the possibility of equivalent representations not conjugate in $G$. This proves (1).

By Corollary 1.2 and the remarks above, $k(M) \leq O\left(q^{3 r^{1 / 2}}\right)$ for each possible $M$.

Thus, $\bigcup_{M \in \mathcal{S}_{4}(G)} M$ is the union of at most $O\left(r^{3 / 2} q^{3 r^{1 / 2}} p^{3 r^{1 / 2}}\right)$ conjugacy classes of $G$. Now apply Theorem 6.15 to conclude that (2) holds.

Putting the previous results together completes the proof of Theorem 7.7 . Theorem 1.5] also follows immediately from the previous results.

\section{REFERENCES}

[A1] Andrews, G., The theory of partitions, Addison-Wesley, Reading, Mass., 1976. MR0557013 (58:27738)

[A2] Andrews, G., Partitions, $q$-series and the Lusztig-Macdonald-Wall conjectures, Invent. Math. 41 (1977), 91-102. MR0446991 (56:5307)

[As] Aschbacher, M., On the maximal subgroups of the finite classical groups, Invent. Math. 76 (1984), 469-514. MR746539 (86a:20054)

[AG] Aschbacher, M. and Guralnick, R., Solvable generation of groups and Sylow subgroups of the lower central series, J. Algebra 77 (1982), 189-201. MR665173 (84c:20025)

[BFH] Benson, D., Feit, W., and Howe, R., Finite linear groups, the Commodore 64, Euler and Sylvester, Amer. Math. Monthly 93 (1986), 717-719. MR863974 (87m:05011)

[Bo] Boston, N., Dabrowski, W., Foguel, T., et al., The proportion of fixed point free elements of a transitive permutation group, Comm. Algebra 21 (1993), 3259-3275. MR1228762 (94e:20002) 
[BW] Britnell, J. and Wildon, M., On the distribution of conjugacy classes between cosets of a finite group, Bull. London Math. Soc. 40 (2008), 897-906. MR2439655 (2009g:20057)

[CC] Cameron, P. and Cohen, A., On the number of fixed point free elements in a permutation group, Discrete Math. 106/107 (1992), 135-138. MR1181907 (93f:20004)

[C] Carter, R., Finite groups of Lie type: Conjugacy classes and complex characters, John Wiley \& Sons, New York, 1985. MR794307 (87d:20060)

[DFG] Diaconis, P., Fulman, J., and Guralnick, R., On fixed points of permutations, J. Algebraic Combin. 28 (2008), 189-218. MR2420785 (2009g:20002)

[D] Dixon, J., Random sets which invariably generate the symmetric group, Discrete Math. 105 (1992), 25-39. MR1180190 (93e:20004)

[FF] Feit, W. and Fine, N. J., Pairs of commuting matrices over a finite field, Duke Math. J. 27 (1960), 91-94. MR0109810(22:695)

[Fe] Feller, W., An introduction to probability theory and its applications, second edition, John Wiley \& Sons, 1957.

[FGS] Fried, M., Guralnick, R. and Saxl, J., Schur covers and Carlitz's conjecture, Israel J. Math. 82 (1993), 157-225. MR 1239049 (94j:12007)

[F1] Fulman, J., Random matrix theory over finite fields, Bull. Amer. Math. Soc. 39 (2002), 51-85. MR1864086 (2002i:60012)

[F2] Fulman, J., Cycle indices for the finite classical groups, J. Group Theory 2 (1999), 251289. MR.1696313 (2001d:20045)

[FG1] Fulman, J. and Guralnick, R., Derangements in simple and primitive groups. In: Groups, Combinatorics, and Geometry (Durham 2001), A. Ivanov et al., Eds., World Scientific, 2003. MR1994962 (2004e:20003)

[FG2] Fulman, J. and Guralnick, R., Derangements in finite classical groups for actions related to extension field and imprimitive subgroups, preprint.

[FG3] Fulman, J. and Guralnick, R., Derangements in finite classical groups for subspace actions, preprint.

[FG4] Fulman, J. and Guralnick, R., The probability of generating an irreducible subgroup, preprint.

[FG5] Fulman, J. and Guralnick, R., Conjugacy classes in orthogonal and symplectic groups, preprint.

[FNP] Fulman, J., Neumann, P.M. and Praeger, C.E., A generating function approach to the enumeration of matrices in finite classical groups, Mem. Amer. Math. Soc. 176 (2005), no. 830. MR.2145026 (2006b:05125)

[Ga] Gallagher, P., The number of conjugacy classes of a finite group, Math. Z. 118 (1970), 175-179. MR0276318 (43:2065)

[Gl] Gluck, D., Characters and random walks on finite classical groups, Adv. Math. 129 (1997), 46-72. MR.1458412 (98g:20027)

[GLS] Gorenstein, D., Lyons, R. and Solomon, R., The classification of the finite simple groups. Number 3, American Mathematical Society, Providence, RI, 1998. MR.1490581 $(98 \mathrm{j}: 20011)$

[GV] Gow, R. and Vinroot, C. R., Extending real-valued characters of finite general linear and unitary groups on elements related to regular unipotents, J. Group Theory 11 (2008), 299-331. MR2419003 (2009c:20082)

[Gu] Guralnick, R., Intersections of conjugacy classes and subgroups of algebraic groups, Proc. Amer. Math. Soc. 135 (2007), 689-693. MR.2262864 (2007i:20071)

[GKS] Guralnick, R., Kantor, W. and Saxl, J., The probability of generating a classical group, Comm. Algebra 22 (1994), 1395-1402. MR1261266 (95a:20030)

[GLMS] Guralnick, R, Liebeck, M., Macpherson, D. and Seitz, G., Modules for algebraic groups with finitely many orbits on subspaces, J. Algebra 196 (1997), 211-250. MR1474171 (98j:20058)

[GMS] Guralnick, R., Müller, P. and Saxl, J., The rational function analogue of a question of Schur and exceptionality of permutation representations, Mem. Amer. Math. Soc. 162 (2003), no. 773. MR1955160 (2004d:12005)

[GR] Guralnick, R. and Robinson, G., On the commuting probability in finite groups, $J$. Algebra 300 (2006), 509-528. MR2228209 (2007g:60011)

[GT1] Guralnick, R. and Tiep, P., The non-coprime $k(G V)$ problem, J. Algebra 293 (2005), 185-242. MR2173972 (2006g:20018) 
[GT2] Guralnick, R. and Tiep, P., Symmetric powers and a problem of Kollár and Larsen, Invent. Math. 174 (2008), 505-554. MR2453600(2009j:20065)

[GW] Guralnick, R. and Wan, D., Bounds for fixed point free elements in a transitive group and applications to curves over finite fields, Israel J. Math. 101 (1997), 255-287. MR1484879 (98j:12002)

[Hu] Humphreys, J., Conjugacy classes in semisimple algebraic groups, Mathematical Surveys and Monographs, 43, American Math. Society, Providence, 1995. MR1343976 (97i:20057)

[I] Isaacs, I. M., Characters of $\pi$-separable groups, J. Algebra 86 (1984), 98-128. MR.727371 (85h:20012)

[J] Jantzen, J., Representations of algebraic groups, second edition, Mathematical Surveys and Monographs, 107, American Mathematical Society, Providence, RI, 2003. MR 2015057(2004h:20061)

[K] Kawanaka, N., On the irreducible characters of the finite unitary groups, J. Math. Soc. Japan 29 (1977), 425-449. MR0450383(56:8678)

[KL] Kleidman, P. and Liebeck, M., The subgroup structure of the finite classical groups, London Mathematical Society Lecture Note Series, 129, Cambridge University Press, Cambridge, 1990. MR1057341 (91g:20001)

[KR] Kovács, L. and Robinson, G., On the number of conjugacy classes of a finite group, $J$. Algebra 160 (1993), 441-460. MR1244923(94m:20057)

[LMS] Liebeck, M., Martin B., and Shalev, A., On conjugacy classes of maximal subgroups of finite simple groups, and a related zeta function. Duke Math. J. 128 (2005), 541-557. MR.2145743(2006b:20043)

[LOST] Liebeck, M., O'Brien, E., Shalev, A., and Tiep, P., On the Ore conjecture, J. European Math. Soc. 12 (2010), 939-1008. MR2654085 (2011e:20016)

[LiP] Liebeck, M. and Pyber, L., Upper bounds for the number of conjugacy classes of a finite group, J. Algebra 198 (1997), 538-562. MR1489911 (99c:20023)

[LPS] Liebeck, M., Pyber, L. and Shalev, A., On a conjecture of G. E. Wall, J. Algebra 317 (2007), 184-197. MR2360145 (2009b:20048)

[LSe1] Liebeck, M. and Seitz, G., Unipotent and nilpotent classes in simple algebraic groups and Lie algebras, preprint.

[LS1] Liebeck, M. and Shalev, A., Fuchsian groups, finite simple groups and representation varieties, Invent. Math. 159 (2005), 317-367. MR2116277 (2005j:20065)

[LS2] Liebeck, M. and Shalev, A, Character degrees and random walks in finite groups of Lie type, Proc. London Math. Soc. 90 (2005), 61-86. MR2107038 (2006h:20016)

[LS3] Liebeck, M. and Shalev, A., Maximal subgroups of symmetric groups, J. Combin. Theory Ser. A 75 (1996), 341-352. MR.1401008 (98b:20005)

[Lu1] Lübeck, F., Small degree representations of finite Chevalley groups in defining characteristic, LMS J. Comput. Math. 4 (2001), 135-169. MR1901354 (2003e:20013)

[Lu2] Lübeck, F., http://www.math.rwth-aachen.de/ Frank.Luebeck/.

[LuP] Łuczak, T. and Pyber, L., On random generation of the symmetric group, Combin. Probab. Comput. 2 (1993), 505-512. MR.1264722 (95b:20004)

[Lus1] Lusztig, G., On the finiteness of the number of unipotent classes, Invent. Math. 34 (1976), 201-213. MR0419635 (54:7653)

[Lus2] Lusztig, G., Irreducible representations of the finite classical groups, Invent. Math 43 (1977), 125-176. MR0463275 (57:3228)

[M1] Macdonald, I., Numbers of conjugacy classes in some finite classical groups, Bull. Austral. Math. Soc. 23 (1981), 23-48. MR615131 (82j:20092)

[M2] Macdonald, I., Symmetric functions and Hall polynomials, second edition, Clarendon Press, Oxford, 1995. MR1354144 (96h:05207)

[Mar] Maróti, A., Bounding the number of conjugacy classes in a permutation group, J. Group Theory 8 (2005), 273-289. MR2137971 (2006e:20054)

[MR] Maslen, D. and Rockmore, D., Separation of variables and the computation of Fourier transforms on finite groups. I, J. Amer. Math. Soc. 10 (1997), 169-214. MR1396896 (97i:20019)

[NP] Neumann, P.M. and Praeger, C.E., Cyclic matrices over finite fields, J. London Math. Soc. 52 (1995), 263-284. MR:1356142 (96j:15017)

[O] Odlyzko, A.M., Asymptotic enumeration methods, Chapter 22 in Handbook of Combinatorics, Volume 2. MIT Press and Elsevier, 1995. MR1373678 (97b:05012) 
[R] Robinson, G., Bounding numbers and heights of characters in p-constrained groups. In: Finite Groups 2003, 307-317, Walter de Gruyter GmbH \& Co. KG, Berlin, 2004. MR2125082 (2006e:20017)

[S1] Seitz, G., Generation of finite groups of Lie type, Trans. Amer. Math. Soc. 271 (1982), 351-407. MR654839 (83h:20021)

[Se] Serre, J.-P., On a theorem of Jordan, Bull. Amer. Math. Soc. 40 (2003), 429-440. MR 1997347(2004f:11040)

[Sh] Shalev, A., Word maps, conjugacy classes, and a non-commutative Waring-type theorem, Annals Math. 170 (2009), 1383-1416. MR2600876

[S] Shintani, T., Two remarks on irreducible characters of finite general linear groups, $J$. Math. Soc. Japan 28 (1976), 396-414. MR0414730 (54:2825)

[St1] Steinberg, R., Endomorphisms of linear algebraic groups, Mem. Amer. Math. Soc., No. 80, 1968. MR0230728 (37:6288)

[St2] Steinberg, R., Lectures on Chevalley groups, Notes prepared by John Faulkner and Robert Wilson, Yale University, New Haven, Conn., 1968. MR0466335 (57:6215)

[T] Tiep, P., Finite groups admitting grassmannian 4-designs, J. Algebra 306 (2006), 227243. MR 2271581 (2007j:20008)

[VW] van Lint, J.H. and Wilson, R.M., A Course in Combinatorics, Cambridge University Press, Cambridge, England, 1992. MR.1207813 (94g:05003)

[W] Wall, G. E., On the conjugacy classes in the unitary, symplectic, and orthogonal groups, J. Aust. Math. Soc. 3 (1963), 1-63. MR0150210 (27:212)

[W2] Wall, G. E., Some applications of the Eulerian functions of a finite group, J. Aust. Math. Soc. 2 (1961), 35-59. MR0125156 (23:A2461)

Department of Mathematics, University of Southern California, Los Angeles, CalIFORNIA 90089-2532

E-mail address: fulman@usc.edu

Department of Mathematics, University of Southern California, Los Angeles, CalIFORNIA 90089-2532

E-mail address: guralnic@usc.edu 